\title{
A Survey of Adaptive Services to Cope with Dynamics in Wireless Self-Organizing Networks
}

\author{
Cigdem Sengul, TU-Berlin/Deutsche Telekom Labs, Germany \\ Aline Carneiro Viana, INRIA, France \\ Artur Ziviani, National Laboratory for Scientific Computing (LNCC), Brazil
}

\begin{abstract}
In this paper, we consider different types of wireless networks that benefit from-and, in certain cases, require-self-organization. Taking mobile ad hoc, wireless sensor, wireless mesh, and delay-tolerant networks as examples of Wireless Self-Organizing Networks (WSONs), we identify that common challenges these networks face are mainly due to lack of centralized management, device heterogeneity, unreliable wireless communication, mobility, resource constraints, or the need to support different traffic types. In this context, we survey several adaptive services proposed to handle these challenges. In particular, we group the adaptive services as core services and network-level services. By categorizing different types of services that handle adaptation and the types of adaptations, we intend to provide useful design guidelines for achieving self-organizing behavior in network protocols. Finally, we discuss open research problems to encourage the design of novel protocols for WSONs.
\end{abstract}

Categories and Subject Descriptors: A.1 [Introductory and Survey]; C.2.1 [Computer Communication Networks]: Network Architecture and Design-Network topology, Network communications, Wireless Communication; C.2.2 [Network Protocols]

General Terms: Algorithms, Design

Additional Key Words and Phrases: Adaptive Networking Services, Store and Forward Networks, Distributed Networks, Wireless Sensor Networks, Delay Tolerant Networks.

\section{INTRODUCTION}

Currently, we witness a proliferation of wireless computing and communication devices. These include a variety of laptops, hand-helds, mobile phones, sensors, connected via multiple wireless technologies, and come with a multitude of applications, data, and services. This rich diversity of the wireless communication landscape requires changing the way we think about networking: Wireless networks increasingly need to self-organize and adapt to changing conditions to ease management and operation ${ }^{1}$. Although self-organization can be defined in many different ways, we follow [Prehofer and Bettstetter 2005] in defining it as "the emergence of system-wide adaptive structure and functionality from simple local interactions between individual entities" and

\footnotetext{
${ }^{1}$ Although some types of wired networks also require and benefit from self-organization, in this work, we only focus on wireless networks.

This work is partially supported by the Brazilian Funding Agencies FAPERJ and CNPq.

Author's addresses: C. Sengul (cigdem.sengul@telekom.de), Deutsche Telekom AG, Laboratories ErnstReuter-Platz 7, 10587 Berlin, Germany; A. C. Viana (Aline.Viana@inria.fr), INRIA Saclay - le de France, Parc Orsay Universit, 4, rue Jacques Monod, 91893 Orsay Cedex, France; A. Ziviani (ziviani@lncc.br), National Laboratory for Scientific Computing (LNCC), Computer Science Department, Av. Getúlio Vargas, 333 - Quitandinha, 25651-075 - Petrópolis - Brazil.

Permission to make digital or hard copies of part or all of this work for personal or classroom use is granted without fee provided that copies are not made or distributed for profit or commercial advantage and that copies show this notice on the first page or initial screen of a display along with the full citation. Copyrights for components of this work owned by others than ACM must be honored. Abstracting with credit is permitted. To copy otherwise, to republish, to post on servers, to redistribute to lists, or to use any component of this work in other works requires prior specific permission and/or a fee. Permissions may be requested from Publications Dept., ACM, Inc., 2 Penn Plaza, Suite 701, New York, NY 10121-0701 USA, fax +1 (212) 869-0481, or permissions@acm.org.

(c) 2011 ACM $0360-0300 / 2011 / 12-A R T 1 \$ 10.00$
}

DOI 10.1145/0000000.0000000 http://doi.acm.org/10.1145/0000000.0000000 
we discuss this concept in the context of wireless networks. Specifically, we survey different types of wireless networks that benefit from - and in certain cases, require self-organization.

In this paper, we drive examples from mobile ad hoc, wireless sensor, wireless mesh, and delay-tolerant networks, which have several common denominators in terms of the challenges they pose. These challenges emerge mainly due to lack of centralized management, device heterogeneity, unreliable wireless communication, mobility, resource constraints, or the need to support different traffic types (presented in more detail in Section 2). Due to these dynamics, these wireless networks undergo frequent changes during their lifetime, which sets them apart from traditional networks. It is important to note that while the presence of such dynamics call for self-organization, it also presents critical challenges to perform it.

To become a reality, these so-called Wireless Self-Organizing Networks (WSONs) require intelligent reconfiguration ability. Therefore, research efforts in this area [Burns et al. 2008; Eriksson et al. 2004; Boonma and Suzuki 2007; Amorim et al. 2008] have focused on systems that can respond to the changes in user requirements and operating conditions as well as configure and reconfigure automatically. In particular, autonomic communication research focuses on self-organizing systems that support selfconfiguration, self-healing, self-protection, and self-optimization [Dobson et al. 2006]. Note that such self-organization requires the network to host distributed, scalable, and adaptive services. Essentially, adaptability to changes in the system or environmentalong with robustness and scalability-is a key necessary characteristic, yet not sufficient, for the emergence of self-organizing behavior or structure in the WSONs we consider. In this paper, we identify these adaptive services as core services, which require only node-level operation, and network-level services, which require cooperation from nodes in the network. For instance, following this rationale, we categorize resource management as a core service and routing as a network-level service. A network-level service can, in turn, be built on a core service (e.g., resource-aware routing for the case of routing as a network-level service). Hence, our focus is mainly on services related to the network and lower layers. End-to-end adaptations related to the transport and application layers (for instance, problems related to multimedia transmissions over wireless as well as issues with TCP or other transport protocols) are out of the scope of this paper.

The importance of supporting adaptive services can be illustrated by the number of projects investigating this issue: ANA [ANA 2006], AWARE [AWARE 2006], SOCRATES [SOCRATES 2007], and RUNES [RUNES 2006], to mention a few. To understand the challenges of adaptivity and the role of different network dynamics, consider an example from the RUNES project. In their "Road Tunnel Fire Scenario", a road tunnel, which is not designed for safety, needs to be continuously monitored by RFID tags. Furthermore, sensors that measure temperature, humidity, and some gases feed air quality information to user PDAs. In the scenario, a collision accident between vehicles creates a fire in the tunnel. Many tunnel users are expected to have personal mobile devices and will call the emergency services for help, and some can also query local information systems about the location of safe places in the tunnel. Various sensors in the tunnel collect data and should help assess the situation and possibly guide users to safer places. A possibility might also be to use robots, which can be sent into the tunnel to locate, for instance, poisonous gases. Thus, some means for data dissemination is required (i) to access the data collected by these sensors and (ii) to send control information back to them, for instance, to update what kind of data they should capture. Additionally, since energy scavenging is not possible in such a scenario, special attention must be paid to the energy levels in sensors. Furthermore, although security and privacy are important issues, in such an emergency situation, a 
victim might rather reveal his medical records to have higher rescue priority. This scenario incorporates different dynamics (e.g., device heterogeneity, energy constraints, the need to update how the data is collected, the mobility of robots, changes in security requirements) and clearly illustrates the relevance of distributed adaptive services (e.g., energy management, data collection, routing) for proper wireless network operation.

The goal of this survey is thus to present a comprehensive review of the literature on adaptive services for WSONs. Our aim is to provide a better understanding of the current research on adaptive services by investigating their important design features and providing discussion about the open research issues in the area. In particular, along with the paper roadmap, we define our contributions as follows:

- Based on a discussion on different network types and their involved dynamics, we define a categorization of the adaptive services needed to deal with these challenges as core services and network-level services (Section 2).

- We then survey the adaptive services found in the WSON literature as core services (Section 3) and network-level services (Section 4). The detailed taxonomy of different services are presented at each related section. Based on these discussions, we intend to provide useful design guidelines for achieving adaptive behavior in network protocols for WSONs.

- Finally, we discuss some related topics that were not covered in this survey, including some new inter-disciplinary techniques to assist self-adaptation. We conclude with an overview of new challenges in adaptive service design (i.e., cross-layer adaptation, simultaneous adaptation to different metrics) and practical implications (Section 5).

\section{SELF-ORGANIZING NETWORKS AND ADAPTIVE SERVICES}

Self-organization can be defined as "the spontaneous creation of a globally coherent pattern out of local interactions" [Heylighen 2001]. Hence, every self-organizing system needs the ability to adapt; in other words, the ability to find a fit between its operation and the environment [Heylighen 2001]. Such adaptivity allows a network to operate more efficiently and predictably under a broader range of varying conditions. Consequently, adaptivity provides robustness and resilience. Therefore, selforganizing networks are expected to be more insensitive to perturbations or faults, and be able to recover fast from the effects of network dynamics. The remainder of this section details different types of self-organizing networks and the involved network dynamics. We also present a categorization for adaptive services, classifying what type of services adapt to changes, when, and how frequently.

\subsection{Network Types and Dynamics}

Wireless networks have a multitude of variable features, which are briefly introduced in Section 1. In this section, we first give a more detailed overview of different wireless networks and their corresponding applications, and second, underline essential network dynamics that affect the operation of these networks.

We focus mainly on wireless ad hoc networks as potential WSONs. The main idea behind ad hoc networks is building "networks anytime and anywhere" without any need for pre-existing infrastructure. The absence of fixed infrastructure means that the nodes communicate directly with one another in a peer-to-peer fashion and provide themselves the basic communication services, such as routing, address allocation, and name resolution. Wireless ad hoc network applications cover various areas, such as military or post-disaster rescue operations, temporary group collaboration at conferences or lectures, and sensor networks. In the following, we give more details on some example networks: 
Table I. The intensity of each network dynamic in a given type of network

\begin{tabular}{|l|c|c|c|c|}
\hline & $\begin{array}{c}\text { Ad hoc networks } \\
\text { (in general) }\end{array}$ & WSN & WMN & DTN \\
\hline \hline Mobility & High & Low & Low to High & Very high \\
Resource constraints & High & Very High & Low to High & Medium \\
Variance in node cooperation & High & Medium & Low to High & High \\
Heterogeneity & High & Medium & Low to High & Very high \\
\hline
\end{tabular}

- Wireless Sensor Networks (WSNs): WSNs consist of small nodes with sensing, computation, and wireless communications capabilities. Typical uses of sensor networks are environmental monitoring and target detection. Hence, the majority of the time, the communication is one-to-all (i.e., sink to sensors) and many-to-one (i.e., sensors to sink). These networks are typically considered homogeneous as a single deployment typically consists of the same type of sensor devices. However, exceptions are the cases where more capable and potentially mobile devices are used as sinks. Therefore, we consider these networks to have medium heterogeneity. It is generally assumed that WSNs are large-scale (e.g., hundreds or thousands of nodes). Finally, sensor nodes are strictly resource-constrained (e.g., in terms of energy, computation capacity, or memory). In particular, mechanisms for optimizing energy consumption constitute an important requirement. However, it must be noted that such mechanisms may affect node cooperation in the network, which operates in a cooperative manner by default. For instance, as nodes drain their batteries, they might refrain from participating in certain network functions, such as routing.

- Wireless Mesh Networks (WMNs): The architecture of WMNs can be classified as infrastructure WMNs, client WMNs, and hybrid WMNs [Akyildiz et al. 2005]. In infrastructure WMNs, mesh nodes form a minimal backbone which typically provides Internet access to their clients. Since these networks are considered to be deployed by one party, the expected heterogeneity and the variance in node cooperation are low. Essentially, these WMNs can be considered as a first step towards providing highbandwidth communication over a specific coverage area. These networks typically need to scale to a high number of clients and to satisfy a diverse set of connectivity needs. Client WMNs allow forming peer-to-peer networks among client machines, and can be considered to inherit the general challenges of "ad hoc networks". On the other hand, in hybrid WMNS, both client-to-client and client-to-infrastructure meshing become possible, relieving some of the challenges such as high mobility and high heterogeneity.

- Delay Tolerant Networks (DTNs): This is a specific class of networks in which connectivity between neighboring nodes is intermittent due to either mobility or the varying conditions of the wireless medium. Examples of DTNs include disaster response, underwater sensor, pocket-switched, and vehicular networks. In such networks, a contemporaneous routing path may never exist between a sender and a receiver. In the literature, this type of network is also referred to as intermittently-connected, or highly-partitioned. Furthermore, DTNs are frequently characterized by strict resource constraints (e.g., in terms of memory, CPU, and energy).

In all these networks, the main network dynamics emerge from a variety of sources, such as mobility (that also affects link reliability), resource constraints, lack of centralized management, reliance on node cooperation, high heterogeneity in resources, or resource failures. The typical intensity of these dynamics in the aforementioned networks is summarized in Table I. This is by no means an exhaustive list-for instance, wireless link connectivity might fluctuate not necessarily due to mobility but also due to variations in environmental conditions. However, we focused on the ones that stem from changes in individual node characteristics and behavior. These network dynam- 
ics have a direct impact on robustness, reliability, performance, and lifetime of the services offered by the WSON. For instance, mobility implies a dynamic topology, which, in turn, implies more complexity for location management, topology maintenance, and routing. Another important dynamic is changes in resources; for instance, changes in available energy require taking measures against node depletion. Furthermore, due to energy harvesting [EHF 2009], the energy states are subject to continuous change (a dead node can come back again after some time). Therefore, WSONs require adaptive services that can handle such changes, which is the topic of next section.

\subsection{Adaptive Services}

Adaptivity provides high flexibility under different operating conditions but, poses significant challenges in terms of the design of such services. In this paper, we define a space of adaptation services based on different network dynamics (as described in Section 2.1). We categorize these services as core and network-level services.

We define core services as services that deal with network dynamics at the node level. In this survey, we focus on two particular core services: location management and resource management. Essentially, we consider main attributes of a node as its location and its available resources, which are the main factors that affect its communication capability in the network. Clearly, a static network would not require location updates to maintain network connectivity. Similarly, if all nodes are tethered, have high CPU power, high bandwidth and no memory constraints, then there is also no need for resource management. In contrast, in the networks we consider, context information and external conditions affect nodes and their wireless links and hence, call for adaptive services. For instance, mobile users in a campus environment (i.e., context) may require a location management solution to facilitate communication. Similarly, weather conditions (i.e., external conditions) can degrade wireless links and consequently, available node bandwidth, calling for an adaptive bandwidth management scheme. Therefore, in this paper, we focus on location and resource management services as core services as they allow nodes to monitor and adapt to changing local conditions to continue their participation to the network.

As a second category, we define network-level services as services that require node collaboration and thus, adapt to changes on how nodes relate to each other. For instance, routing and data collection fall under this category. Note that network-level services can also be built on core services. In Sections 3 and 4, we give detailed examples of core and network-level services, respectively.

Additional categorizations are also possible. Services can be categorized based on whether they need implicit or explicit feedback [Gross et al. 1999]. Services that work with implicit feedback require a built-in functionality of passive monitoring and deriving information about the current conditions. For instance, a node can monitor the data traffic of its neighbors and based on the received signal strength in the monitored period, make decisions about whether its neighbors are moving or not. On the other hand, services that rely on explicit feedback require external support for providing such information in a pull/push-based manner. For instance, to monitor its neighborhood, a node might collect periodic hello messages from its neighbors and depending on these messages make decisions about neighbors' presence. Based on our example, a node relying only on passive monitoring might conclude wrongly that a neighbor has moved away, while the node is still in the given neighborhood but currently not sending any data. Hence, implicit feedback can only provide local and less accurate information through passive monitoring, which further might be difficult to interpret. On the other hand, although explicit feedback provides more accurate information, it has the disadvantage of higher overhead as well as requiring some sort of external support (e.g., nodes should know they should be sending periodic hello messages). 


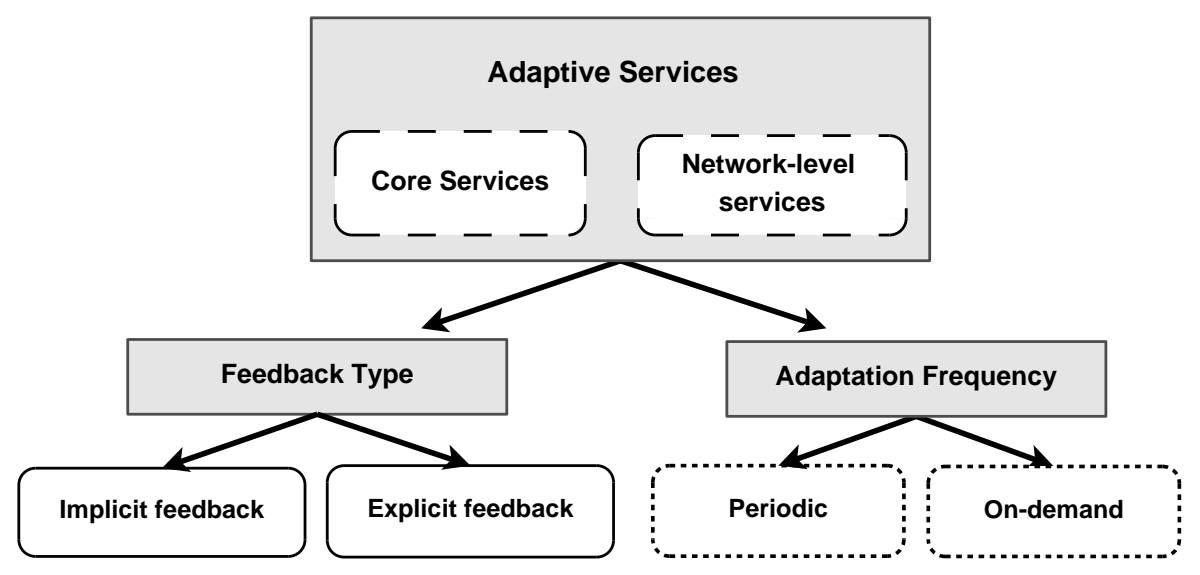

Fig. 1. Categorization of adaptive services.

Furthermore, we can categorize services onto ones that adapt periodically and ones that adapt on-demand [Gross et al. 1999]. A periodic adaptive service regularly assesses the operating conditions and adapts if needed. In contrast, an on-demand adaptive service may trigger some changes when a performance parameter drops below a value or when more resources are discovered, characterizing an event-driven adaptivity to varying conditions.

Figure 1 depicts our categorization for adaptive services. In the remainder of this paper, we build on this categorization to survey and classify adaptive services for WSONs in the literature. In each respective section, we depict the taxanomy of a service based on these different types of classification and also relate to which type of networks these services are most appropriate based on the example networks in Section 2.1.

\section{CORE SERVICES}

We see core services as services that need to be present given a network dynamic. For instance, if node locations are dynamic, a location management service can be considered as a core service on which nodes can register/update their locations or query locations of other nodes. Several network-level services can be built upon this core service, where routing, topology management, and data dissemination are the most straightforward examples. Additionally, core services can also be seen as node-level services, where nodes govern their operation based on changes. For instance, if node's energy goes lower than a certain level, it might choose to stop forwarding messages for other nodes. Thus, energy management, or more generally, resource management stands out as another core service. In this section, we present these two core services-location management in Section 3.1 and resource management in Section 3.2-and discuss in detail their operation based on the categorization for adaptive services presented in Section 2.

\subsection{Location Management}

Knowing the physical location of a node from an identifier of that node, such as a name or IP address, enables a whole class of location-aware applications or services in WSONs. Examples of location-aware applications or services are: position-based filtering of content availability and monitored data, navigation orientation, sending messages to nodes within a certain region (i.e., geocasting), and geographic routing. In WSONs, mobility is the key characteristic that affects the location of nodes. Therefore, 
location management is the key to adapt the network behavior in face of changing conditions due to mobility.

Before discussing location management in WSONs, we first establish a difference between positioning systems and location services. A positioning system allows a user or device to know its own position, providing the basic information for each node to locate itself in space. Such a position may be indicated either through an absolute location (e.g. outdoors positioning system such as the Global Positioning System (GPS) [Enge and Misra 1999] and indoors positioning systems such as Active Badge [Harter and Hopper 1994] and RADAR [Bahl and Padmanabhan 2000]) or relative location (e.g. virtual coordinate systems such as Vivaldi [Dabek et al. 2004] or the ones proposed by [Kermarrec et al. 2009]) In short, by using a positioning system each node is able to know its own location. Location may be represented in many forms depending on the application, such as positions on an absolute or relative coordinate system as well as context-aware location information (e.g. office 502). Different from positioning systems, location services provide the location of other nodes. Therefore, based on the information acquired through a positioning system, nodes announce their locations using a location service to allow other nodes to locate them.

The spontaneous nature of WSONs requires a dynamic association between identification and the location of a node, as well as the specification of a mechanism to manage this association, i.e., a location service. Providing a scalable and efficient location service in the context of WSONs is a difficult problem. The idea of decoupling location and identification contrasts with the current overloaded semantics of IP addressing. In general, when a source wants to communicate with a destination, the only information it has is the destination's identifier. The location service is responsible for translating this identifier into an address that describes the destination's location in the network. The resulting structure intends to deal with the shortcomings of IP addressing, thus allowing a more flexible and dynamic structure capable of better adapting to changes in location due to mobility.

We classify the adaptive location management approaches as in Figure 2. It must be noted that the type of approaches we discuss are targeted to connected mobile networks and hence, will not necessarily apply to DTNs where disruptions might partition the network for long periods of time. Dissemination-based approaches distribute the location information throughout the network in an unstructured way, whereas rendezvousbased approaches rely on specific points to publish the location information of each node in the network. These are further discussed in the following.

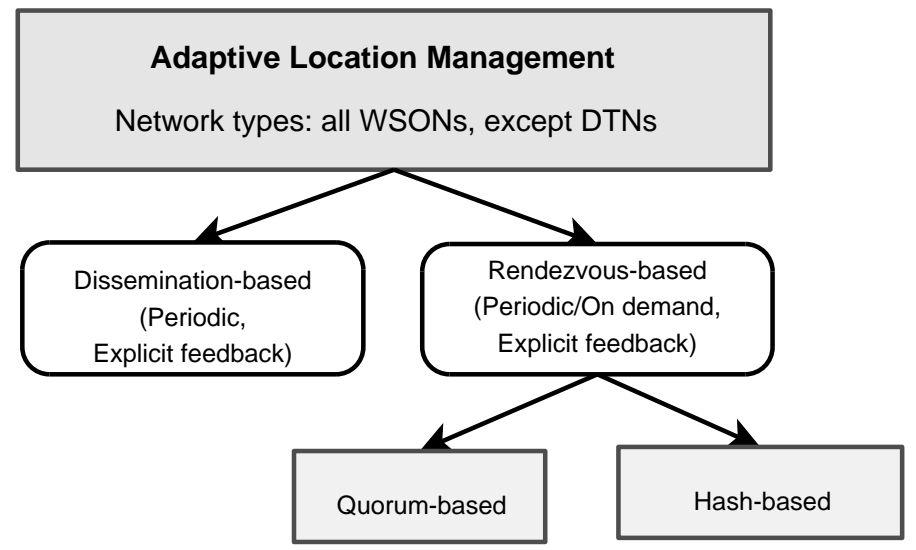

Fig. 2. Taxonomy of adaptive location management approaches. 
3.1.1. Dissemination-based approaches. The simplest dissemination approach relies on the straightforward concept of each node flooding the network with its location information. Nevertheless, this simple alternative clearly does not scale and is thus inefficient in highly dynamic network scenarios. Alternatives to simple flooding rely on the use of a dissemination model to distribute the location information throughout the network. For instance, nodes' location information could be replicated throughout the network during packet forwarding as nodes move. The location knowledge is thus based on the distributed estimations of node positions in the network [Grossglauser and Vetterli 2003; Dubois-Ferriere et al. 2003; Benbadis et al. 2005]. Nevertheless, evaluations of these algorithms [Ioannidis and Marbach 2005] show that their performance is closely tied to the nodes' mobility process and the density of neighbors along the path of the destination and along the path of a packet moving towards the destination. This dependency on the mobility level of the network makes these algorithms less adaptable to highly dynamic WSONs.

3.1.2. Rendezvous-based approaches. Some approaches in the literature consider nodes in a region as rendezvous points, which maintain the location information of other nodes. For instance, in the Terminode project [Hubaux et al. 2001], each node advertises its current position to a geographical region called Virtual Home Region (VHR) with a fixed center $C_{V H R}$ and a variable radius $r$. This fixed center is determined by each node by hashing its identifier with a publicly known hash function. Nodes further inform their region about their current location as they move, in order to always remain reachable by other nodes. Approaches like the Terminodes are also known as home region location services [Giordano and Hami 1999; Woo and Singh 2001; Li et al. 2000]. In this context, satisfactory location query performance (e.g., high query success rate, low round-trip query latency) can be achieved by using multiple home regions for each node [Cheng et al. 2002; Seet et al. 2005; Li et al. 2000]. In particular, [Seet et al. 2005] adopt a demand-driven approach in creating and maintaining multiple home regions in order to reduce the communication overhead caused by location updates. The proposed approach is based on the actual demand for each node and the locality of the querying sources. In particular, whenever there is a demand for the location of a node and the querying nodes are not in the primary region of the node, secondary home regions are temporarily created close to the querying nodes.

Rendezvous-based approaches may be further categorized by the way node identifiers are mapped onto the rendezvous points within the network. Typical ways of performing such a mapping are based either on quorums or distributed hash tables (DHTs), which are discussed next. The interested reader may find additional surveys focused particularly on location services in [Stojmenovic 2002; Kiess et al. 2004], while a performance comparison of some location services can be found in [Das et al. 2005].

Quorum-based. Quorums are used in update and search queries [Liu et al. 2008; Haas and Liang 1999]: a node updates its position by sending the information to a subset of nodes (update quorum); a source node requests the location of a destination from another subset of nodes (query quorum). The update and query quorums must be designed properly to have common nodes.

Hash-based. Examples of self-organizing systems built upon the functionalities of DHT are: Peernet [Eriksson et al. 2004], Terminodes [Hubaux et al. 2001], GLS [Li et al. 2000], Twins [Viana et al. 2006], DLM [Xue et al. 2001], and SOLIST [Busnel et al. 2008; Busnel et al. 2008]. These proposals basically differ in the way the DHT-based location service is implemented. Their main idea is to use a hash function to distribute location information among rendezvous points throughout the net- 
work. This hash function is also used by a source to identify the rendezvous point that stores a destination's location information. Changes in nodes' location can be thus easily dealt by updating rendezvous points, which allows, in turn, DHT-based routing protocols [Viana et al. 2005] to adapt to different levels of node mobility.

\subsection{Resource Management}

Resource management that encompasses a broad range of limited resources - such as CPU, bandwidth, memory, and energy - in the presence of network dynamics is a very challenging problem. To address this problem, one approach is to model each node as a self-interested agent that tries to maximize its profit by selling goods (e.g., sensor readings in WSNs, or forwarding services) subject to budgets, for instance, energy budgets [Mainland et al. 2005]. Using reinforcement learning, nodes react to network dynamics and the changes in their budgets. Different trade-offs can be achieved by setting prices based on, for instance, lifetime, data fidelity, and latency. In the current literature, however, the majority of resource adaptive approaches focus on one particular resource, among which energy and bandwidth are the most commonly studied. Therefore, in the remainder of this section, we will focus on adaptive energy management and adaptive bandwidth management.

3.2.1. Adaptive Energy Management. In many examples of resource management approaches, energy stands out as one of the most critical resources, as it depends on the remaining battery of the node and availability of recharging. Energy management in WSONs is mainly focused on the energy consumption of the wireless network interface as it is one of the main consumers. The network interface expends energy for both communication and idling. Hence, approaches to energy management target at reducing one or both of these costs. To reduce communication costs, power and rate control are typical approaches, which allow to transmit with just enough power rather the maximum power and with the highest rate possible. However, the main challenge stems from the need to continuously adapt to the current channel conditions so that the right transmission power and rate can be set.

On the other hand, to reduce idling costs, the typical approach is to introduce a duty cycle, which switches nodes to a low-power sleep mode. However, during the sleep mode, nodes are not able to receive or transmit and therefore communication performance (both in terms of throughput and latency) might be impaired. Recent studies show that fixed periodic duty cycles neither can save energy efficiently nor can accommodate network traffic effectively [Zheng and Kravets 2003]. Therefore, adaptive duty cycling based on current network conditions becomes a must. Motivated by these observations, we categorize the current adaptive approaches to energy management as (i) adaptive communication and (ii) adaptive sleeping. In the remainder of this subsection, these approaches are discussed in detail. Figure 3 summarizes the categorization of the approaches in this subsection. Note that these approaches can generally apply all types of WSONs. A few exceptions are when a specific network application is used to gain further energy savings, which will be discussed later in this section.

Adaptive Communication. Communication energy is affected by two factors: (i) the transmission power level and (ii) the time spent in communication. While transmission power control (TPC) is a very well known energy-saving technique to reduce (i), rate control approaches save from (ii). However, it must be noticed that since TPC affects the signal strength, it also affects the choice of bandpass modulation schemes and rates. Hence, TPC and rate control need to work together to achieve expected energy savings. Therefore, in this section, we will focus on works that discuss TPC and rate control jointly and leave the discussion about pure rate control protocols for bandwidth management to 3.2.2. 


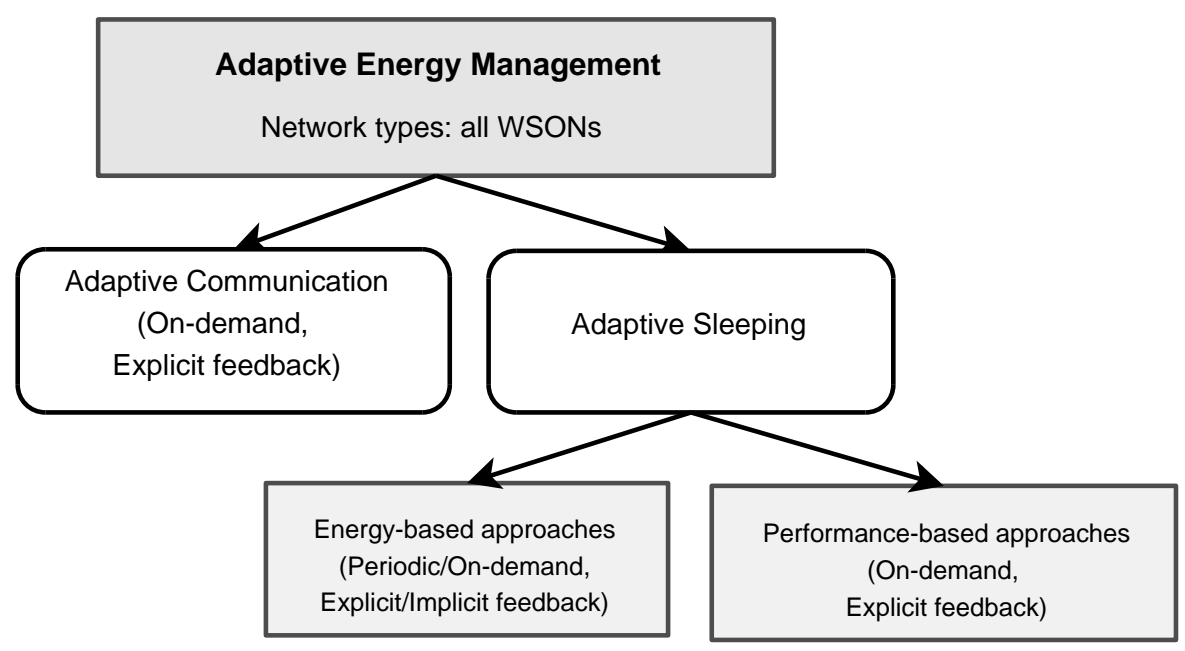

Fig. 3. Taxonomy of adaptive energy management approaches.

TPC affects the network in multiple ways. For instance, by selecting power levels among nodes carefully, the topology of the network can be controlled to satisfy certain characteristics (e.g., a k-connected network, a broadcast tree). We leave the discussion on such adaptive approaches that actually deal with topology management to Section 4.1. Furthermore, TPC affects the signal strength and so, the level of interference among nodes. Hence, spatial reuse (i.e., the capability to accommodate concurrent transmissions) can be improved by adequate power adjustment. This aspect of TPC is out of the scope of this section, and for a thorough discussion of the subject, we refer the interested readers to [Krunz et al. 2004]. The main focus of this subsection is rather the effect of TPC on transmission energy.

The majority of the TPC and rate control approaches require transmit power information from the sender (i.e., explicit feedback) and adapt to the current conditions on-demand. For instance, assuming a convex relationship between rate and transmission energy (i.e., energy decreases with slower rates) lazy scheduling of packets was proposed [Uysal-Biyikoglu et al. 2002; Kompella and Snoeren 2003]. More specifically, in [Kompella and Snoeren 2003], each node estimates the current demand on the wireless channel and adjusts the transmission schedules accordingly based on the convex rate-power relationship. Based on similar assumptions, [Schurgers and Srivastava 2002] propose an adaptive bit loading approach, which sets the modulation level based solely on the current channel conditions so that energy consumption is minimized. However, note that choosing a bandpass modulation scheme with a slower data rate increases the amount of time for transmission. In [Harris 2006], it is shown that although slower rates may result in a lower transmission power, the amount of energy used to drive the transmitter during this time can outweigh these savings. Therefore, the proposed approach, Pincher, first uses information about the SNR (signal to noise ratio) at the receiver to determine the bandpass modulation scheme with the fastest possible rate. It then uses TPC to match the chosen bandpass modulation scheme. By choosing the fastest data rate and minimizing the time spent in transmit mode, it becomes possible to save more energy.

Adaptive Sleeping. The main challenge to adaptive sleeping is to find a duty cycle that fits the current network traffic: sleep when there is no traffic but be immediately available when traffic is present [Sengul et al. 2007]. With fixed duty cycling ap- 
proaches (e.g., IEEE 802.11 PSM [IEEE 802.11 WG ]), network throughput is shown to degrade under heavy traffic [Zheng and Kravets 2003], while under light traffic, energy is wasted in idling. Additionally, latency grows linearly with the number of hops as each intermediate node has to wait for the receiver to wake up before it can forward a message [Ye et al. 2002] (the so-called "data forwarding interruption problem" [Lu et al. 2007]). Furthermore, as nodes drain their batteries, they may need to switch to sleeping more aggressively and stay in this state longer. Therefore, the input for adaptive sleeping approaches can be categorized as: (i) current network traffic, which is determined by the application and communication model; and (ii) remaining energy, which is determined by the battery model and the existence of energy harvesting techniques. Depending on the priority given to each input information, we further categorize the existing body of work as performance-based and energy-based approaches.

- Performance-based approaches: The main idea behind these approaches is to match the duty cycling to network traffic as much as possible. Note that some multi-radio solutions also exist, which use a low-power wake-up radio to turn on the high-power data radio at the exact time when the data radio is needed [Schurgers et al. 2002; Sengul et al. 2008]. However, these approaches are out of the scope of this paper as they cannot be considered adaptive solutions. In adaptive approaches, either the senders shape the duty cycle of the receivers depending on their own traffic [van Dam and Langendoen 2003; Zheng et al. 2003] or the receivers try to predict future messages and stay active to accommodate the network traffic [Hu and Hou 2004]. For the sender-based approach (explicit feedback), receivers are typically kept awake by "future request-to-send" messages [van Dam and Langendoen 2003; Zheng et al. 2005; Zheng et al. 2003; Lu et al. 2007]. For the receiver-based approach (implicit feedback), a node stays active as long as it observes "activation events", such as reception of data or acknowledgment (ACK) messages or simply by sensing communication on the channel [van Dam and Langendoen 2003; Zheng and Kravets 2003]. In [Hu and Hou 2004], potential receivers try to predict the future traffic using overheard ACK messages and hence, keep awake. Using these predictions, LISP opens a "data freeway" so that communication proceeds with minimum delay. These approaches all adapt on-demand based on the existing traffic.

Complementary to staying awake longer to serve network traffic, a node might also adapt to silent periods and hence, sleep longer. For instance, P-MAC [Zheng et al. 2005 ] tunes the duty cycles based on a node's own and its neighbors' traffic so that nodes can increase their sleep times in the presence of low traffic. Longer sleep times is also possible through low-power listening protocols, which use long preambles to ensure that the receiver is awake at the time of the transmission [Polastre et al. 2004]. Observing such a scheme would be penalized during busy periods, SCPMAC [Ye et al. 2006] is proposed as an adaptive solution, which switches nodes between low-power listening and duty cycling depending on the current traffic load. Different than other approaches that are agnostic to network applications, the duty cycles can also be better fine-tuned using application-specific information. For instance, D-MAC [Lu et al. 2007] targets a data collection application in sensor networks. In these networks, typically, an aggregation tree is built and using this information, duty cycles of nodes in the tree can be staggered to address the "data forwarding interruption problem". Further benefits can also be obtained by using "data prediction", which lets parent nodes to receive messages from multiple children without wasting an active cycle. Similarly, BSD (Bounded-Slowdown) protocol [Krashinsky and Balakrishnan 2005] tunes the sleep schedules accordingly to Web traffic, where small round trip times and short connections are expected. 
Most importantly, realizing the need to match active/sleep duty cycles to current traffic led to efforts for standardization. Although targeted to infrastructure-based wireless networks, IEEE 802.16e standard [IEEE 802.16e-2005 and IEEE Std 802.162004/Cor1-2005 ] describes a sleep mode with 3 different "Power Saving Classes". While for best-effort or non-real time variable-rate traffic, nodes are allowed to double their sleep times, for real-time traffic, nodes follow fixed sleep/active duty cycles. A third power-saving class is targeted to periodic control traffic (i.e., the case when the base station knows when the next message will be sent). However, the truly adaptive behavior, which chooses the power saving class type for each connection, still remains to be addressed by the standard.

-Energy-based approaches: The goal of energy-based approaches is to save energy at every opportunity. On the contrary to, and perhaps also complementary to, performance-based approaches that shape duty cycles based on traffic, energy-based approaches use traffic shaping based on duty cycles [Poellabauer and Schwan 2004; Havinga and Smit 2001]. Basically, these approaches allow the network to be accessed in bursts, and hence, reduce the number of state transitions. Note that state transitions consume energy due to device switching overhead as well as causing protocol-level "data forwarding interruption problem" [Lu et al. 2007]. Hence, in these approaches, the goal is to buffer packets as long as possible before their deadlines, so that a burst of packets can be sent reducing energy consumption. Since the main consideration is traffic deadlines, these approaches fall onto the on-demand and explicit feedback category.

Clearly, the energy-based approaches operate more sensitive to available energy at nodes compared to the performance-based approaches. The goal is to keep nodes alive as long as possible, with limited focus on the effects on performance. Therefore, which kind of approach is more beneficial still remains as an open research problem.

3.2.2. Adaptive Bandwidth Management. Unlike the wired networks, where bandwidth is abundant, bandwidth in WSONs is scarce. Furthermore, the available bandwidth changes in time based on the context and traffic conditions, namely, mobility, weather conditions, network heterogeneity and due to internal interference from competing traffic inside the network or external interference from other networks [Shah et al. 2005; Xu et al. 2003; Mirhakkak et al. 2001].

In this section, we will look at two different approaches to handle these changes. The first approach is automatic rate control that increases or decreases transmission rates based on observed link quality [Bicket 2005]. This type approach is applicable to all types of WSONs. The second approach is the more general bandwidth control, which tries to infer the current available bandwidth to be used in upper-layer services such as admission-control or congestion control. This type of approach generally assumes a connected network, where bandwidth information can be disseminated periodically. Therefore, its applicability to DTNs is limited. Fig. 4 summarizes the categorization of the approaches in this subsection.

The first auto-rate protocol to exploit multi-rate capability at the physical layer was ARF (Auto Rate Fallback) [Kamerman and Monteban 1997]. ARF uses higher transmission rates after consecutive transmission successes and reverts to lower rates after failures (i.e., implicit feedback, and on-demand adaptation). An extension, adaptive ARF algorithm [Lacage et al. 2004], increases the number of consecutive successes necessary to go to a higher rate after each failure. In another approach, RBAR (Receiver-Based Auto Rate) [Holland et al. 2001], the receivers explicitly set the transmission rate to be used for the next packet using specialized RTS (Request-to-Send) and CTS (Clear-to-Send) message exchange (i.e., explicit feedback, on-demand adaptation). However, wireless links may exhibit significantly varying properties and there- 


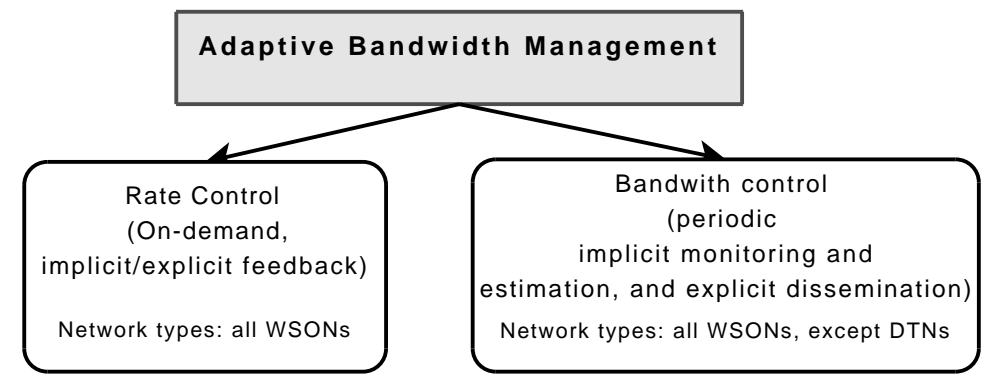

Fig. 4. Taxonomy of adaptive bandwidth management approaches.

fore, RSSI is not always indicative of link quality. Furthermore, in some real networks, it was shown that for some links a lower rate performs worse than a higher rate [Bicket 2005]. Based on these observations, SampleRate [Bicket 2005] allows probing other rates than the current rate and switches to another bit rate if the estimated perpacket transmission time becomes smaller. OAR (Opportunistic Auto Rate) [Sadeghi et al. 2005] takes a different approach, where the nodes that experience high channel quality, and hence, higher transmission rates are allowed to send more packets back-to-back under the constraint that the temporal-share of the channel is equivalent among neighboring nodes. In other words, with OAR, if a node is able to transmit at $11 \mathrm{Mbps}$, it will be allowed to transmit more packets and obtain close to 5 times more throughput compared to a node transmitting at $2 \mathrm{Mbps}$. It must be noted that in this case, both nodes still access the channel approximately $50 \%$ of the time. Since nodes are informed by how long a node will access the channel (i.e., More Fragments bit is set in the MAC header), this approach is considered an on-demand explicit feedback approach.

Regardless of the algorithm used, the changes in transmission rates translate to changes in available bandwidth. Furthermore, due to shared nature of the wireless medium, it is important to take into account not only a node's own transmissions but the transmissions of all the nodes in the neighborhood. Finally, the extent of external interference (e.g., from other networks) also determine a node's effective available bandwidth capacity.

Bandwidth control mechanisms try to take these changes into account to enable QoS provisioning in WSONs. In [Shah et al. 2005], the rate used by the MAC layer is directly fed back to the upper layers to indicate the changes in the node's available bandwidth. In [Xu et al. 2003], bandwidth management component directly observes the channel utilization ratio to measure the available bandwidth (i.e., using implicit feedback) in a periodic manner. The channel utilization ratio is calculated by observing the channel state changes from busy to idle and from idle to busy. For a given time period $T$, the channel utilization rate for the period $t, R_{t}$, is channel_busy_period $/ T$. Nodes maintain an EWMA (Exponential Weighted Moving Average) of $R_{t}$ to smooth the channel utilization rates and estimate the bandwidth as $B W_{t} \approx W\left(1-R_{t}\right)$, where $W$ is the raw channel bandwidth based on the transmission rate of the radio (i.e., 54 Mbps in IEEE 802.11a).

On the other hand, in [Shah et al. 2005], the available bandwidth is estimated by looking at the current throughput, $T P=S /\left(t_{r}-t_{s}\right)$, where $S$ is the size of the packet, $t_{s}$ is the the time the packet is ready at the MAC layer, and $t_{r}$ is the time that an ACK has been received. The time interval $t_{r}-t_{s}$ captures channel contention and retransmissions and hence, can help estimate the current link conditions. Hence, the 
bandwidth of a link is measured implicitly and in a periodic manner in discrete time intervals by averaging the throughput of recent packets in the past time window.

In [Xu et al. 2003], bandwidth estimations are propagated pro-actively or retrieved on demand by the routing mechanism. This information is used by a call admission control mechanism. Once flows are admitted to the network, flows are regulated by a congestion control mechanism that uses information again from the adaptive bandwidth management component. In [Shah et al. 2005], the bandwidth requirements at application/middleware layer are translated to channel time proportion (CPT) requirements at the MAC layer. Given the bandwidth estimations and requirements, admission control and flow rate adaptor mechanisms are used to regulate traffic in a single-hop wireless network. In both works, adaptive bandwidth control plays a key role as a core service for both admission control and congestion control.

\section{NETWORK-LEVEL SERVICES}

We consider network-level services as services that require collaboration from nodes to perform a specific task for the network. The network-level services typically rely on the core services presented in Section 3. The services included in our work are roughly categorized as topology management, routing and forwarding, and data management. In the rest of this section, for each network-level service, we define its basic operation and what kind of adaptations become possible given the network dynamics.

\subsection{Topology Management}

Topology management can be defined as shaping the network topology to achieve a certain objective, such as better connectivity, fault-tolerance, or energy management. The network topology can be modified (i) through node adaptation (e.g., by imposing a hierarchy in the network through clustering or removing nodes from the network graph by powering nodes off); (ii) through link adaptation (e.g., using transmit power control, smart directional antennas, or intelligent channel assignment); and (iii) controlled mobility (e.g., in actuator sensor networks), which affects both the presence of nodes and links, as well as the quality of links in the network graph. In the remainder of this subsection, we span these techniques, which are also summarized in Figure 5 including the type of networks they can be used in.

4.1.1. Topology management through node adaptation. The topology of a network can be most easily modified by imposing a hierarchy over the network through clustering. In general, such a clustering process allows better address assignment, bandwidth utilization, and energy consumption, as most communication remains local. For instance, given a wireless sensor network, the LEACH protocol [Heinzelman et al. 2000] rotates cluster-heads periodically so that one node collects all local information and transmits to the base station. Since the selection of cluster-heads affects how the data is transmitted in the network, we also mention this approach under data dissemination protocols, in Section 4.3.2. Similarly, in the B-protocol [Basagni et al. 2001], a small subset of the network nodes form a backbone in a mobile ad hoc network to serve other nodes (e.g., help routing) based on their current status determined by mobility, node/link failures, and energy resources. This type of protocol can also be seen as a hybrid routing protocol, which we discuss in Section 4.2.1 and where more examples can be found.

The majority of the topology control techniques that power nodes off have similar goals: to control the network density by turning off nodes for energy savings while preserving the forwarding capacity of the network. These approaches operate similarly to the cluster-based approaches. However, nodes that are chosen to be on always remain awake and do not use a sleep scheduling scheme to exploit the absence of traffic (refer 


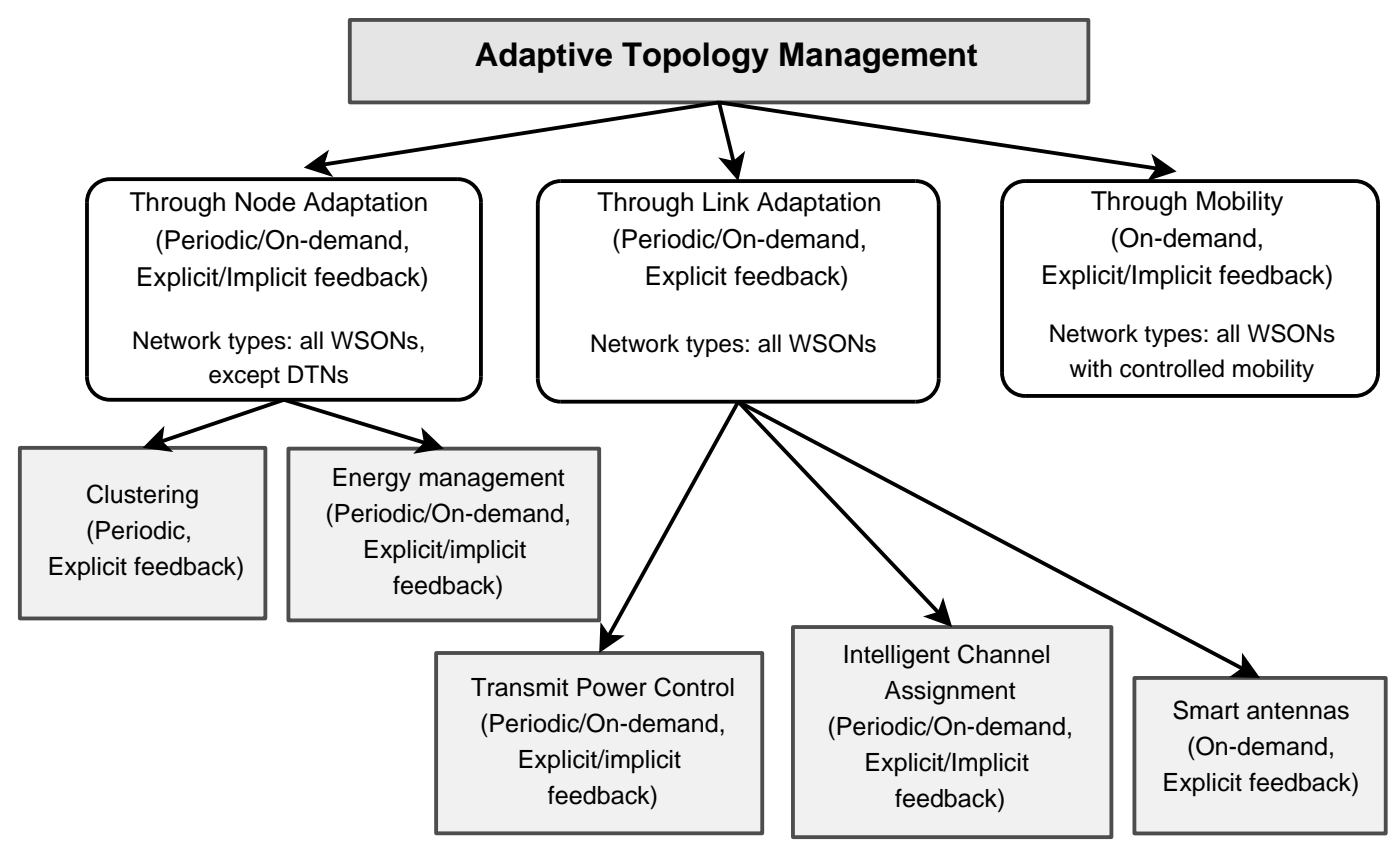

Fig. 5. Taxonomy of adaptive topology management approaches.

to Section 3.2.1). AFECA [Xu et al. 2000] maintains a constant density of active nodes by periodically turning radios off for an amount of time proportional to the measured number of neighbors of a node (i.e., implicit feedback, periodic approach). Hence, as network regions become more crowded, more energy can be conserved. In SPAN [Chen et al. 2001], nodes make local decisions on whether to sleep or to join a forwarding backbone (i.e., become a coordinator). Each node bases its decision on an estimate of how many of its neighbors will benefit from it being awake, and the amount of energy available to it. SPAN leads to a periodic rotation of coordinators since nodes announce the current conditions via periodic messages (i.e., explicit feedback). Similarly, ASCENT [Cerpa and Estrin 2004] measures local connectivity based on neighbor and packet loss thresholds and nodes join the backbone based on both the current status and the application requirements. TITAN [Sengul and Kravets 2005] builds a backbone on-demand using implicit information from both on-going communication and whether nodes are currently on or off. The design of TITAN is based on the trade-offs between waking up power-saving nodes on shorter routes and using longer routes that contain active nodes. While the aforementioned approaches target at forwarding and routing services, SAND [Merrer et al. 2006] coordinates the node sleep and wakeup cycles for sensing and/or routing states, which is more in tune with the requirements of wireless sensor networks.

4.1.2. Topology management through link adaptation. One of the common techniques to adapt the topology is adding (deleting) links to (from) the network graph through transmission power control. In [Burri et al. 2006], to cope with wireless channel dynamics, each node periodically order its neighbors based on link quality measurements, and exchanges this information with other neighbors. Nodes select links to communicate over based on this information. Additionally, this link selection is also repeated on-demand when a node detects a link quality change. To combat the network dynamics, again a periodic approach is proposed in [Costa et al. 2008], where nodes 
increase their transmission powers until they reach a threshold number of neighbors. In the case of critical nodes, which cannot be covered with this simple scheme, nodes cooperate to increase their transmission power levels to cover these nodes too. Similarly, in ABTC (Ant-Based Topology Control) [Shen et al. 2005], each node periodically collects local information from nearby nodes via sending and receiving ant packets. By using swarm intelligence as an adaptive search mechanism, ABTC finds a good power assignment for the mobility situation based on the minimization objectives of either the total network or maximum node energy consumptions.

While the aforementioned techniques adapt to channel conditions, in [Park and Sivakumar 2003], nodes adapt to current traffic conditions by measuring the local contention time in two-hop neighborhood (mini-channel) and modifying their transmission power based on the current channel utilization status. In the case of over-utilization, a node increases transmission power to enlarge the area of its mini-channel and hence, reduce the number of one-hop flows. In case of under-utilization, a node decreases its transmission power to allow more one-hop flows in its mini-channel. Based on this idea, three different coordination schemes are proposed: (i) ATC-CP, in which all nodes use a common power, (ii) ATC-IP, in which each node independently uses its locally determined transmission power, and (iii) ATC-MS, which employs a one-hop masterslave coordination.

The second technique for link adaptation is intelligent channel assignment, which affects which nodes can hear each other, and hence, the existence of a link between nodes. In [Makram et al. 2008], considering a multi-channel wireless mesh network, a Cluster Channel Assignment (CCA) approach is proposed, where the aggregate throughput is maximized by exploiting spatial reuse and local dynamic switching of channels based on the current traffic load. In [Ramachandran et al. 2006], an interference-aware channel assignment algorithm is presented, where mesh nodes are re-configured periodically so that they operate in a minimum-interference channel assignment. The period itself can also be tuned to reflect how frequently the interference levels in the mesh network are expected to change.

Finally, using multibeam smart antennas, two nodes can communicate if both sending and receiving beams point towards each other and hence, a link exists conditionally between nodes, thus affecting the network topology. In [Rokonuzzaman et al. 2008], the network is initialized with a connected topology, and depending on the current communication and the required QoS, the topology control layer changes the topology by creating directional links between neighbors to optimize the network performance.

4.1.3. Topology management through mobility. In networks using devices with controlled mobility (e.g., sensor actuator networks), the ability to move nodes creates a great potential for adaptivity as the network topology can be changed to meet deployment requirements as well as improve network performance and fault-tolerance. In [Dalu et al. 2008], each node is able to move and makes local decisions to keep connectivity to a selected node and therefore, moves with this particular node to maintain the desired topology. In [Wang et al. 2006], all sensor-actuator nodes execute self-deployment protocols to remove coverage holes in a sensor network, where Voronoi diagrams are used to discover these coverage holes. The authors propose three movement-assisted sensor deployment protocols, which either uses virtual forces (e.g., nodes at a close distance repel each other) or pull-based algorithms to move nodes to their local maximum coverage holes. However, the main principle is moving sensors from densely deployed areas to sparsely deployed areas. In [Wang et al. 2005], this work is extended so that the framework does not rely on flooding for redundant node discovery. Here, redundant nodes are found by a quorum-based location service (see Section 3.1) running over the network grid, and relocated to address node failures or respond to an 


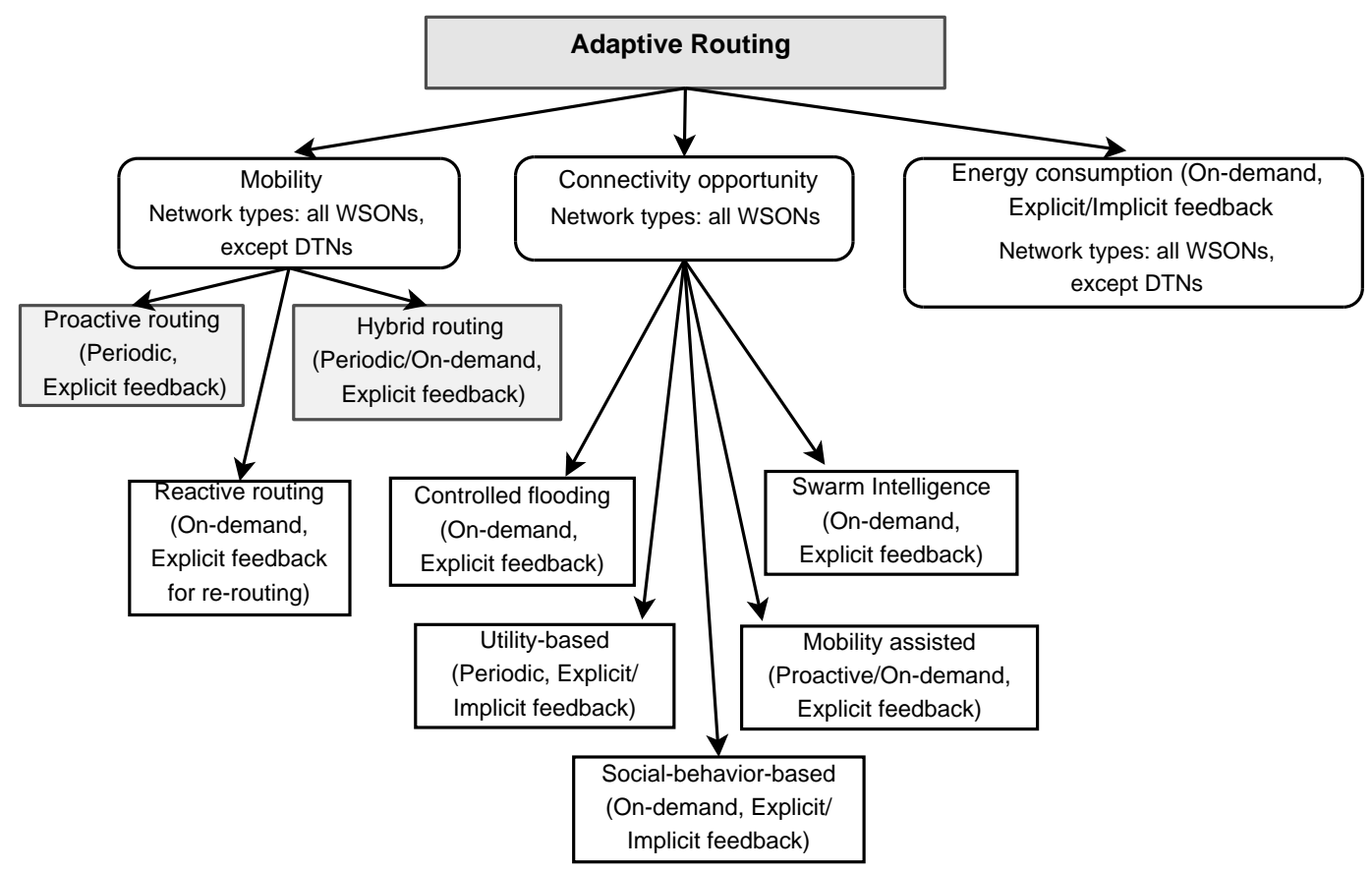

Fig. 6. Taxonomy of adaptive routing approaches.

event that requires additional nodes. While in [Wang et al. 2005], pre-knowledge of the sensor field is used, in [Li et al. 2007], which also uses a grid structure, redundant node information is maintained in local proxies. For instance, to maintain the sensing coverage, these proxies can be queried to choose the right redundant node to relocate. In [Garetto et al. 2008], during deployment, the sensor network is configured to monitor a geographical area with a given node density. However, upon detection of a physical phenomenon, using a virtual force-based strategy, nodes relocate themselves to properly monitor and control the event, while maintaining the network connectivity. As soon as the event ends, nodes return to the monitoring configuration.

In addition to improving coverage in sensor networks, mobility can also be used to improve network performance in more general networks with controlled mobility. In [Burns et al. 2008], some nodes adapt their movement patterns to meet the bandwidth and latency requirements of the network. Using these nodes to deliver datawhich mainly act as 'taxi cabs on call" - compensates the mismatch between available capacity and traffic demands.

\subsection{Routing and Forwarding}

In this section, we discuss adaptive routing and forwarding in terms of the factors that drive the adaptation: mobility, connectivity opportunity in scenarios with intermittent connectivity, and energy consumption. While the approaches based on connectivity opportunity can be applied to all WSONs, the approaches based on mobility and energy consumption typically assume a connected network, and therefore exclude DTNs. This categorization is illustrated in Figure 6.

4.2.1. Mobility. With the advent of the mobile computing, the first main problem to address was to deal with the dynamics introduced by node mobility. As node mobility changes the network topology frequently, specific adaptive solutions needed to be 
designed for network mechanisms and protocols that directly depend on the topology, e.g., routing. In this section, we discuss the main adaptive approaches to mobile routing protocols as well as the main concepts behind them, presenting representative proposals for each approach (which have inspired many variations for different purposes) [Hong et al. 2002; Abolhasan et al. 2004]. It is worth noting that all the approaches discussed at this point are intended for routing or forwarding in a connected wireless network, i.e., where an end-to-end path exists between any two nodes in the network. Later in this section, we will also discuss techniques dealing with intermittently-connected networks.

Proactive Routing. In general, proactive routing protocols periodically distribute routing tables throughout the network. This is intended to maintain fresh lists of possible destinations and routes to them at each node or at least nodes close to these destinations. In this sense, proactive routing is also known as table-driven routing. Scalability is typically addressed by reducing the number of nodes that store and forward global routing information (e.g., using multipoint relaying in OLSR (Optimized Link State Routing Protocol) [Clausen and Jacquet 2003]). Although proactive approaches have the advantage of having the routing information readily available, they could be quite costly to maintain specifically in scenarios of high mobility where topological changes are frequent and may affect a large portion of nodes. As a consequence, the main disadvantages of proactive routing approaches are the potentially high amount of data for maintenance and possibly slow reaction to failures and large topology changes. Well-known examples of adaptive proactive routing protocols are DREAM (Distance Routing Effect Algorithm for Mobility) [Basagni et al. 1998], OLSR [Clausen and Jacquet 2003], and TBRPF (Topology Dissemination based on Reverse-Path Forwarding routing protocol) [Ogier et al. 2004].

Reactive Routing. Reactive routing protocols intend to reduce the control overhead compared to proactive routing by storing information only for active routes. This is done by determining and maintaining routes only for nodes that require to send data towards a specific destination. Routes are typically discovered by a (controlled) flooding of a route request through the network. When a node that has an active route to a destination (or the destination itself) is reached, a route reply is sent back to the source node. Because of the way reactive protocols operate, they are also known as ondemand routing protocols. Although reactive routing has the advantage of lower control overhead, it also presents high latency time in route discovery as a disadvantage. Representative examples of adaptive reactive routing protocols are TORA (Temporally ordered routing algorithm) [Park and Corson 1997], LAR (Location Aided Routing) [Ko and Vaidya 2000], AODV (Ad hoc On-Demand Distance Vector) [Perkins et al. 2003], and DSR (Dynamic Source Routing) [Johnson et al. 2007].

Hybrid Routing. Hybrid routing protocols intend to combine the advantages of proactive and reactive routing. In general, the main idea is to group nearby nodes, which proactively maintain routes. The routes to nodes located outside a group are determined using on-demand route discovery. Most hybrid approaches partition the network in location-based zones. Nodes could also be grouped into trees or clusters based on different optimization criteria, such as improving topology management (see Section 4.1.1). Hybrid routing protocols tend to yield higher scalability compared to pure reactive or proactive protocols because the overheads of the pure approaches are controlled better. However, to achieve such scalability, the main challenge in hybrid protocols is to find a good balance between the number of groups and the size of each group, thus operating in a sweet spot in terms of overhead of intra-group proactive routing and inter-group reactive routing. Examples of hybrid routing approaches are 
DDR (Distributed Dynamic Routing) [Nikaein et al. 2000], SLURP (Scalable Location Update Routing Protocol) [Woo and Singh 2001], and ZRP (Zone Routing Protocol) [Haas et al. 2002].

4.2.2. Connectivity opportunity. Recently, there has been a growing interest in the networking community for reliable routing solutions in wireless networks that face problems such as connectivity problems. Different scenarios encompass disaster response networks, interconnecting sensors monitoring animal habits, or simply providing connectivity to users that carry their portable devices from one connectivity island to another (i.e., pocket-switched networks). In the literature, these kind of networks have been commonly referred to as delay-tolerant, intermittently-connected or highlypartitioned [Fall 2004]. In these networks, communication is extremely challenging as forwarding paths may be unstable and reachability may be highly variable. Therefore, there is a need for robustness against intermittent connectivity, and hence, delay tolerance. Clearly, intermittent network connectivity, where disconnections could be long-lived, changes the network communication problem drastically. Another type of network that tries profit from connectivity opportunities but in an heterogeneous environment is the so-called OppNets (Opportunistic networks) [Lilien et al. 2006; Lilien et al. 2007]. OppNets constitute a category of ad hoc heterogeneous networks that self-configure themselves and try to detect "foreign" devices or systems, which might join the network dynamically using all types of communication media (e.g., Bluetooth, wired Internet, WiFi, ham radio, RFID, satellite) [Lilien et al. 2006; Lilien et al. 2006]. In the following, we focus our attention to the main forwarding approaches that meet the challenges of delay-tolerant networks. However, note that these approaches can be used in general WSONs as well.

- Controlled flooding approaches: Epidemic routing is a well-known technique for data forwarding, which has the goal of maximizing message delivery and latency with a trade-off in resource consumption [Vahdat and Becker 2000]. In epidemic routing, a node buffers messages and passes them on to all the new nodes it contacts as it moves. Hence, messages are eventually delivered to their destinations. Epidemic routing is simple and provides high reliability under changing conditions, but it is not adaptive and hence, might generate too many redundant messages in lossy networks, wasting communication and battery resources. On the other hand, if each node knew the contact probability, waiting times [Francois and Leduc 2006; Jain et al. 2004], buffer limits, and traffic demands [Jain et al. 2004], optimal routing strategies could be computed. However, as these are not typically available, one approach is to disseminate similar information to the entire network [Ramanathan et al. 2007; Su et al. 2006]. For instance, PREP [Ramanathan et al. 2007] uses link availability to drop packets that have lower chance of delivery. In [Su et al. 2006], a link-state protocol is used to maintain information about the expected delay to each node in the network. Based on a minimum path search on the link-state tables, a node transmits a message to a contact if the message would experience less delay through this contact. -Utility-based approaches: Utility-based approaches intend to reduce the costs of previously discussed approaches by selecting a few, but qualified relays. In this context, several approaches estimate a delivery likelihood based on how often contacts meete.g., MaxProp [Burgess et al. 2006], PROPHET [Lindgren et al. 2003], Delegation Forwarding [Erramilli et al. 2008], and Spray and Focus [Spyropoulos et al. 2007]. These approaches might be too conservative and lose suitable forwarding opportunities in environments with scarce connectivity. A less conservative utility-based approach that optimizes resource consumption is [Balasubramanian et al. 2007]: at each forwarding opportunity, nodes evaluate if the gain from replicating a message justifies the resources used. Note that, such an evaluation requires a control channel 
and relies on information about the replicas of a message in the entire network (i.e., the number and location of packet replicas). Authors in [Krifa et al. 2008] propose a mechanism to locally estimate the total number of replicas and then to decide which messages to replicate or drop to achieve either minimum delay or maximum delivery ratio. In this case, nodes need to maintain a history of each message forwarded by each node they meet.

- Social-behavior-based approaches: There is a large amount of recent research devoted to understanding human mobility patterns [Chaintreau et al. 2007; Conan et al. 2007; Lindgren et al. 2006; Mtibaa et al. 2006; Cai and Eun 2008] and social network properties [Hui et al. 2008; Borrel et al. 2008; Daly and Haahr 2007] in DTNs. For instance, a recent study [Chaintreau et al. 2007] shows that the aggregate inter-contact times follow a power-low distribution, which might lead to infinite delays for some forwarding approaches. In contrast,[Conan et al. 2007] gives a more optimistic result, where pairwise inter-contact times are found to be exponentially or log-normally distributed. Empirical evidence from some mobility traces also shows that contacts with friends are more valuable than strangers [Su et al. 2004], indicating there is a bias among different contacts. Therefore, the knowledge of contact patterns is expected to be beneficial for improving forwarding performance.

In [Sengul et al. 2009], contact history, which contains observations both on contact and communication patterns, is used to make predictions about future meetings and thus, consequently, to make forwarding decisions. BUBBLE [Hui et al. 2008] and SimBet [Daly and Haahr 2007] use information about social community structures and popularity within a community to choose more suitable relays. Essentially, using such quality information is beneficial for improving the communication, although it does not always improve reliability [Erramilli et al. 2008; Spyropoulos et al. 2008].

- Mobility-assisted approaches: The idea of using mobility to increase capacity and to provide communication services for nodes in sparsely connected networks is not new [Gupta and Kumar 2000; Jea et al. 2005; Zhao et al. 2004; Glance and Snowdon 2001]. The Data Mule [Jea et al. 2005], Message Ferrying [Zhao et al. 2004], or Pollen [Glance and Snowdon 2001] schemes make use of mobility to improve capacity and connectivity. Data Mules and Message Ferries are mobile nodes that can provide "connectivity opportunities" by moving around the deployment area and by taking responsibility for carrying data between nodes or groups of nodes. Unlike Data Mules, Message Ferries are specialized nodes whose trajectories are known. More specifically, authors in [Zhao et al. 2004] investigate both 'node-initiated' mobility, where the nodes move to meet a known message ferry trajectory, or 'ferry-initiated' mobility, where the nodes send communication requests via a long range radio, and the message ferry moves to meet them. Pollen, however, proposes to use people to carry messages between devices and between physical places.

More recently, the concept of Throwboxes [Zhao et al. 2006] has been proposed as specialized nodes, similar to Data Mules and Message Ferries, to increase capacity in sparsely connected networks by increasing contact opportunities among nodes. However, unlike Data Mules and Message Ferries, Throwboxes are static nodes that function essentially like postal mailboxes, where mobile nodes passing by can simply drop data. Throwboxes decide which node should get which message in order to increase the likelihood of delivery to the intended destination.

- Swarm intelligence-based approaches: Swarm intelligence approaches allow each flow to use its own optimization criteria based on its QoS requirements rather than using a single metric for all flows [Gelenbe 2009]. For instance, especially in frequently disrupted networks, ant colony algorithms are expected to adapt better as all packets are autonomous and follow the paths with stronger pheromones [Kassabalidis et al. 2001]. However, discovered routes might not always be optimal. On 
the other hand, Cognitive Packet Networks (CPNs) [Gelenbe et al. 2001] use smart packets to discover paths based on some QoS criteria, and dumb packets to only carry data over previously discovered paths. Hence, while better routes could be found in $\mathrm{CPNs}$, the reaction to changes would be slower.

4.2.3. Energy consumption. The final category we consider are routing protocols that use energy management as a core service (see Section 3.2). One example that uses power control as a core service is PARO [Gomez and Campbell 2003], where any node can inject itself to the route, if this route optimization results in reductions in transmission power. However, note that PARO model uses an ideal radio model, hence only considers transmit power as an optimization criterion, assuming the transmission energy dominates the energy consumed in other radio modes (e.g., idle mode). Nevertheless, such radios currently do not exist and hence, energy savings from introducing relays between nodes that can transmit directly to each other are rather limited [Gomez and Campbell 2003; Sengul and Kravets 2007]. There also exists several approaches that uses sleep schedules and battery status of the nodes in addition to transmission power levels. For instance, in [Kim et al. 2003], routes with minimum drain rate (MDR) are given higher priority, where the drain rate is the predicted lifetime of a node as a function of its current traffic conditions. Another energy-sensitive approach is to switch the routing metric depending on the current energy state. For instance, in Conditional Max Min Capacity Routing (CMMBCR) [Toh 2001], initially minimum energy routes are chosen when nodes have sufficient remaining battery capacity. However, if all nodes are below an energy threshold, capacity-aware routing is used to determine routes. Similarly, the Conditional Minimum Drain Rate (CMDR) protocol [Kim et al. 2003] chooses routes based on minimum transmission power when the lifetime prediction of nodes are higher than a pre-defined threshold and switches to using MDR once nodes fall below this threshold.

\subsection{Data Management}

Data is an important element in any computer network. In general WSONs, but especially in wireless sensor networks, data management turns to be an essential design problem and must address a new collection of challenges (e.g. coordinating actions with other nodes, limited processing speed and storage capacity). In the following, we discuss adaptive algorithms proposed for different tasks related to the data management service:

- Data collection and gathering: Data flows originate in the network and are directed towards an external entity responsible for collecting the data (e.g. gathering of monitored data).

— Data dissemination: Data flows are directed towards the network (e.g. node configuration, distributed data storage).

- Data processing: Data flows are manipulated within the network (e.g. data compression).

This categorization is illustrated in Figure 7 and detailed in the following.

4.3.1. Data collection and gathering. To achieve true adaptivity (and thus selforganization), one approach is to get inspirations from the nature. For this purpose, researchers started focusing on creating biologically-inspired architectures for WSONs [Boonma and Suzuki 2007]. In these systems, data collection agents sense their local network conditions and adaptively invoke behaviors such as pheromone emission, replication, migration, reproduction, and death. For instance, replication allows an agent to task child agents with the same functionality and migrate to a different node. By pheromone emission, an agent announces such migrations or migration 


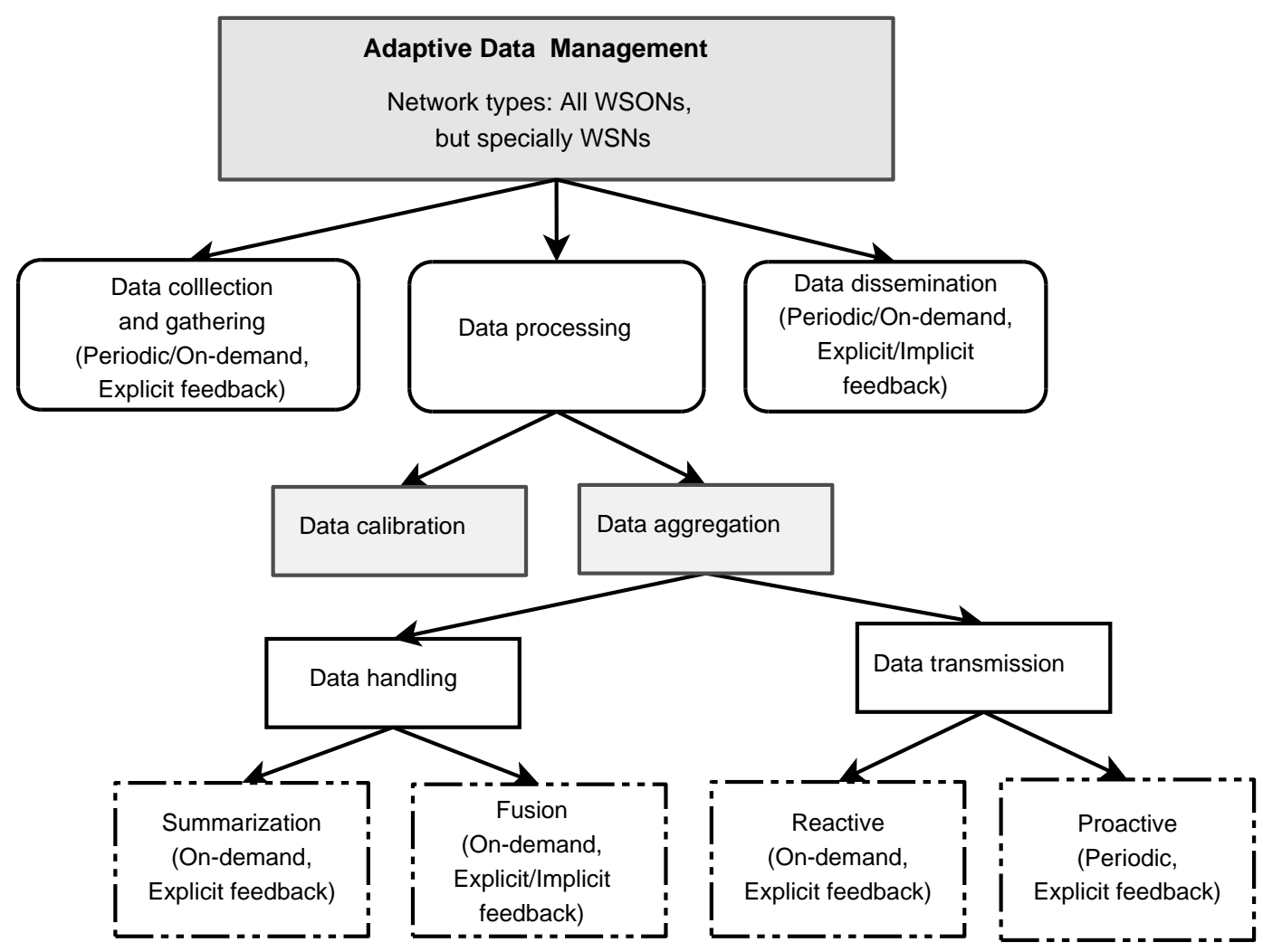

Fig. 7. Taxonomy of adaptive data management approaches.

failures leaving hints for other agents about the good paths to follow. On the other hand, through reproduction, agent behavior evolves with genetic operations (i.e., mutation and crossover). The goal of reproduction is to fit the current environment better to fulfill different, and sometimes conflicting, network objectives such as low latency, low energy consumption, or high delivery success [Boonma and Suzuki 2007; Sengul et al. 2008]. Thus, if an agent follows good policies that find low-latency paths to the target destination, then its genes become more dominant over an agent that consumes too much energy to reach the same destination. Similarly, by invoking death, an agent eliminates useless policies from the network. The system using these adapting agents, MONSOON, was shown to achieve latency, energy, and delivery success objectives even if the network is highly dynamic with new node arrivals, and random and correlated node failures [Boonma and Suzuki 2007].

In the case of WSNs with mobile sinks, data gathering is related to how the mobile sink gathers the data collected by the sensors in the network. In other words, depending on how data is stored in the network, the sink may adapt its trajectory, by either following a predetermined trajectory with controlled mobility (e.g., the sink must visit some predefined nodes to retrieve a representative view of the monitored area) or move freely in the network following an uncontrolled mobility pattern and still harvest a representative view as long as it visits a minimum number of nodes, no matter which one. Therefore, in WSNs with mobile sinks, data dissemination approaches depend on how the sink gathers the data in the network (i.e. the kind of trajectory-controlled or 
uncontrolled) [Chatzigiannakis et al. 2008], and may be characterized as proactive or reactive. Next, we present more details about these strategies.

Reactive strategies for data dissemination with mobile sinks. In reactive strategies, sensors adaptively react to the presence of the mobile sink, by, for instance, receiving a query from it. The sensors then start sending data towards the current position of the sink or to other nodes that are located close to the predicted sink trajectory [Jea et al. 2005; Luo et al. 2006; Basagni et al. 2008]. In this case, the sink must visit some predefined set of nodes to retrieve a representative view of the monitored events or phenomena. On the other hand, reactive dissemination approaches where the mobile sink follows a free, i.e., uncontrolled, trajectory, require the sensors to track the sink mobility in order to adapt or influence the data dissemination. Hence, the sink only collects data from sensors along its trajectory or from sensors that route their data towards the sink. Examples of reactive data dissemination with uncontrolled sink mobility are [Urgaonkar and Krishnamachari 2004; Yang et al. 2006; Wang and Wu 2006]. In particular, some authors have investigated approaches that learn (i) the past movement patterns of the sink(s) to predict its future locations or (ii) the node's past success rate of transmitting data packets to the sink to use the fittest nodes as relays to the sink [Wang and Wu 2006].

Proactive strategies for data dissemination with mobile sinks. In proactive strategies, sensors adaptively disseminate their monitored data towards a subset of sensors that play the role of storage nodes. In controlled sink mobility, this subset of nodes typically form a virtual structure within the WSN that make the data available to be retrieved later by the mobile sink. These solutions with a rendezvous virtual structure basically differ on how this structure is built: grids [Luo et al. 2005] or quorums [Friedman et al. 2008], for instance. Additional details can be found in [Hamida and Chelius 2008], a recent survey on proactive data dissemination strategies in WSNs with mobile sinks. On the other hand, no structure can be defined if the mobile sink performs uncontrolled mobility, since no indication is provided a priori about the trajectory of the sink. In this case, the ideal dissemination would be a uniform data distribution throughout the WSN, where adaptive mechanisms (e.g., according to network density, or to distance to the source) would limit the overhead of the propagated messages and still assure reliability. This dissemination allows a sink following an uncontrolled trajectory to retrieve a representative view of the monitored area by visiting a relatively small number of nodes in the network. Some approaches following this goal are proposed in [Viana et al. 2009; Vecchio et al. 2010].

4.3.2. Data Dissemination. Data dissemination determines how data is propagated to destinations or how it is organized in the network in order to be later gathered by a static or mobile entity. In addition, the data might also be pre-processed in the network for this purpose, which is the topic of the next section. A simple mechanism for data dissemination in connected wireless networks is probabilistic flooding, which computes a local probability to rebroadcast a message or not [Drabkin et al. 2007; Tseng et al. 2003]. Authors in [Drabkin et al. 2007] proposed an adaptive probabilistic forwarding to limit the communication overhead in the network. For this, the forwarding probability is set to be inversely proportional to the number of neighbors. Other adaptive mechanisms that can effectively inhibit redundant rebroadcasts while obtaining high reachability and low latency were proposed in [Tseng et al. 2002; Tseng et al. 2003]. These adaptive mechanisms rely on counter-based, distance-based, or location-based broadcasting.

Different than the aforementioned approaches, the family of SPIN protocols (Sensor Protocol for Information via Negotiation) [Heinzelman et al. 1999; Kulik et al. 2002] 
proposes a resource-adaptive protocol and uses data negotiation in order to avoid the waste of communication and energy resources of flooding-based approaches in WSNs with static sinks. Similarly, the (DHT over GPSR)-based data-centric storage [Ratnasamy et al. 2002] proposes a way to support data-centric storage, where a distributed hash table structure is used to map data to the node that will store it. Authors extend their approach to adaptively deal with failure, mobility, and large numbers of events.

4.3.3. Data Processing. Different than general WSONs, one key aspect in WSNs is the processing of data within the network to prepare it for data collection or dissemination. Note that, WSNs are composed of devices, although limited in capability, that can perform sensing, processing using an on-board microprocessor, and wireless communication. Hence, it is natural to design protocols that use localized communications and in-network processing to, for instance, to respond to data queries. Hereafter, we discuss the two types of data processing usually performed in WSNs, namely data calibration and data aggregation.

Data Calibration. Data accuracy is of prime importance some WSNs, since some applications (e.g., battlefield surveillance) require a high level of data accuracy reported by the network. For this reason, researchers started focusing on calibration algorithms, which map raw sensor readings into corrected values by identifying and correcting systematic bias. In particular, without calibration, readings produced by a sensor could range anywhere from having subtle inaccuracies to being completely meaningless, rendering, in turn, storage, forwarding, or aggregation of this data useless [Buonadonna et al. 2005; Bychkovskiy et al. 2003; Balzano and Nowak 2007].

Additionally, data accuracy may be affected by external environmental conditions (e.g. temperature, pressure, and humidity) and/or by internal conditions (e.g., variables such as energy level and available memory). This requires sensor nodes to adapt to such environmental variations to maintain data accuracy in original levels [Avancha et al. 2004; Hu et al. 2007; LaMarca et al. 2002]. For this purpose, some approaches use ontologies to enrich sensor-data description. In these approaches, an ontology is used to better organize sensor information and to assist users and/or search engines in retrieving relevant information [Avancha et al. 2004; Hu et al. 2007]. In particular, authors in [Avancha et al. 2004] propose to organize the network in clusters and to have cluster heads execute a statistical model-based algorithm to dynamically calibrate sensed data based on the current energy level and the state of on-board sensor of each node in the cluster. The algorithm enables to dynamically determine the most appropriate state of operation for the sensor nodes under existing environmental conditions.

In mobile WSNs, in [LaMarca et al. 2002], robots equipped with accurate and calibrated sensors perform adaptive in-place calibration. In addition, predictive models of sensor behavior are used to generate estimated sensor readings, forcing a more timely robotic recalibration if a sensor is suspected to be drifting. The result is an adaptive mechanism in which the tradeoff of sensor accuracy vs. robotic work can be adjusted at will. Authors in [Miluzzo et al. 2008] propose to use opportunistic interaction between calibrated and uncalibrated mobile nodes to solve a discrete average consensus problem, leveraging cooperative control over their sensor readings and adapting to the sensing context of nodes.

Data Aggregation. Considering the classical sensor-to-sink communication in WSNs, data aggregation can be applied to combine data coming from different sources enroute to a sink, allowing in-network consolidation of redundant data [Croce et al. 2008]. Data aggregation can be done in two precision levels: node and/or network level. At a node level, data aggregation is performed by data handling [Kimura and Latifi 2005]; while 
at the network level, the aggregation requires data transmission over the network. These two approaches are strongly correlated.

- Data handling: The process of data handling can be classified as data summarization and data fusion. Data summarization constitutes an interesting example of the correlation between data manipulation and transmission. It is typically carried out by partially answering a received request at enroute nodes in the path to the issuer [Jelasity et al. 2005; Massoulie et al. 2006; Talebi et al. 2006]. It can be characterized by the use of (i) simple operators: such as maximum or minimum value requests (ii) complex operators: such as average or counting requests. In this context, a time adaptive weighting method to improve convergence behavior of distributed consensus averaging schemes is proposed in [Talebi et al. 2006]. Authors in [Jelasity et al. 2005] introduce an adaptive gossip-based communication protocol for calculating aggregates (e.g., average, minimum, maximum, counting) in a proactive manner. By adaptive, they mean that if the aggregate changes due to network dynamics or variations in the input values, the output of the aggregation protocol will track these changes reasonably quickly.

On the other hand, data fusion is performed by nodes that compress collected and/or received data in order to optimize the use of the available memory. Data compression eliminates or reduces data redundancy, reducing thus future transmissions toward sink nodes. It can be classified into lossless-more adapted to critical applications demanding high accuracy, which can not tolerate measurement corruptions due to the compression process [Marcelloni and Vecchio 2008; 2009; Barr and Asanovic 2006; Sadler and Martonosi 2006]—or lossy —applied when only a summary or a resulting value of monitored events is required. The appropriate type of compression depends on the kind of application, datasets, and/or users concerned. In [Luo et al. 2006; Luo et al. 2005], authors present an adaptive fusion-driven routing protocol that not only optimizes routed information, but also embeds the decisions as to when and where a fusion shall be performed. A lossy wavelets-based compression is proposed in [Ganesan et al. 2002; Chen and Fowler 2005] where compression adapts to the correlation in the data (e.g., temporal, spatial) in different regions [Ganesan et al. 2002] or to the required accuracy [Chen and Fowler 2005]. Correlation is exploited to reduce dimensionality and can vary over time depending on the changing characteristics of the sensed field.

- Data transmission aggregation: The data aggregation by transmission reduction is performed by eliminating the data redundancy only. We classified current work as reactive approaches-where queries are pushed all the way to the remote sensors [Kulik et al. 2002; Hellerstein et al. 2003; Intanagonwiwat et al. 2000]—and proactive approaches, where useful sensor data is pushed from the sensors independently of query reception [Ratnasamy et al. 2002; Heinzelman et al. 2000]. Data transmission aggregation is tightly related to energy and routing management (see Sections 3.2 and 4.2 , respectively) as well as data dissemination and collection.

Aggregation is typically performed with the goal of either (i) minimizing energy consumption, by reducing the energy expended by the sensors during the process of data gathering [Estrin et al. 1999; Intanagonwiwat et al. 2000; Heinzelman et al. 1999]; or (ii) maximizing the network lifetime [Xue et al. 2005; Heinzelman et al. 2000]. TAG [Hellerstein et al. 2003] executes aggregation queries in a time and energyefficient manner by using an isobar mapping approach as the aggregation technique. The main objective of isobar mapping is to build a topographic (contour) map of a space populated by sensors, adapting thus to the deployed region. Aggregation in Directed Diffusion [Estrin et al. 1999; Intanagonwiwat et al. 2000] is performed as data is routed from sensors to the requester, i.e., the sink. Data follows a reverse 
high-quality delivery path with an associated gradient, which matches the interest disseminated by the sink. Isoline aggregation [Solis and Obraczka 2005] targets spatially and temporally-correlated data and groups nodes that report similar readings into isoclusters. LEACH [Heinzelman et al. 2000] is a clustering-based protocol for data transmission aggregation toward the base station. Its main features include adaptive local coordination for cluster formation among sensors, randomized rotation of cluster heads for improved energy utilization, and local data compression at cluster heads to reduce the amount of data being sent from clusters to the base station. Note that as LEACH [Heinzelman et al. 2000] imposes a hierarchy over nodes in a given network topology, we also include it under topology management through node adaption, in Section 4.1.1.

\section{DISCUSSION AND OUTLOOK}

In this survey, we sample a large spectrum of adaptive services (i.e., routing, resource, topology, location, and data management) targeted to deal with the dynamic nature of different types of Wireless Self-Organizing Networks (WSONs), such as wireless sensor, mesh, and delay tolerant networks. Accordingly, in this last section, we perform an overall discussion on the general limitations of coping with this dynamic nature of WSONs taking into account the surveyed proposals so far. We also discuss the limitations of this survey due to its bounded scope. While we present a broad coverage of the current state-of-the-art, this survey paper is not meant to be exhaustive. Other topics of interest are, for instance, security as an adaptive network service, and new types of networks, such as cognitive radio networks. Cognitive Radio Networks (CRNs) are particularly interesting, as they are composed of fully programmable cognitive wireless devices that can sense their environment and dynamically adapt their channel access method, spectrum use, and networking protocols as needed for suitable network and application performance [Akyildiz et al. 2009; Mitola and Maguire 1999]. The goal is to provide opportunistic spectrum usage for interoperability of totally or partially disconnected networks [Gorcin and Arslan 2008; Majid and Ahmed 2008; Younis et al. 2009; Zhang et al. 2006], as well as adapt channel access based on primary users and secondary cognitive users. Additionally, mobile cognitive radio devices might be empowered with self-deploying features, supporting deployment according to the location of remaining devices of the affected network and the geographic conditions of the region.

Also related to adaptive communication and interoperability in heterogeneous networks, the attention of the networking research community has recently turned to the Future Internet. This Next-Generation Internet will likely interconnect a much wider variety of devices and will also be highly heterogeneous in terms of the types of networks it interconnects. In fact, the vision is that there will be a "wired" core interconnecting network "clouds" that gravitate at the edges. These clouds could be wired or wireless, infrastructure-based or not, deployed impromptu or in a planned way. Once deployed, these clouds might need to self-organize forming a network of interconnected devices.

Similarly, as the mobile telecommunication industry moves towards next-generation $4 G$ systems, the ability to automate, especially the management processes, has emerged as a key technology requirement. The idea is to achieve self-configuring, selfoptimizing networks, which, for instance, allows auto-configuration of new base stations at site, as well as automatic reconfiguration of live networks, instead of having to manually tune the network each time a small change is required.

As seen from these examples, the present state of the affairs hints loudly at the current and increasing need for adaptive solutions. Moreover, differently than the majority of the works presented in this paper, the new adaptive approaches should con- 
sider multiple optimization criteria and more complex variabilities in the system (e.g., both mobility and energy level changes). We believe, as the research moves towards more complex problem formulations, more interdisciplinary approaches will emerge. We have already presented some examples, such as swarm intelligence for data routing and forwarding, but we believe the WSON research community will continue to be inspired by social structures, biology, physics, games, and control systems.

While adaptive operation will indeed be a necessity, one must take care that the complexity introduced to the system should not outweigh the gains achieved. First, it must be noted that not every functionality should be adaptive-adaptivity should be applied when it is absolutely necessary. Furthermore, adaptive approaches proposed for different purposes (e.g., mobility approaches vs. data dissemination) should be able to work together and no negative interactions occur. Therefore, holistic system analysis and evaluation will play an important role to understand the true benefits of adaptive systems for WSONs. Currently, this is a neglected area in adaptive systems research. However, looking at the other side of the coin, care must be taken so that the current technology itself does not limit the design of solutions, for instance, due to hardware, software or communication constraints (e.g., limited processing power and memory, closed firmware, limited bandwidth). Therefore, the network protocol design should follow a development cycle, where adaptivity of each component is considered in terms of both complexity and gain, and then, implemented depending on the current constraints. These components should be constantly re-evaluated based on the obtained improvements on network performance and relaxation of constraints in terms of hardware, software, and communication.

In conclusion, though the early solutions to cope with the dynamic nature of WSONs was initiated by DARPA in 1997 [Huebscher and McCann 2008], the research on adaptive networking services are still increasingly interesting and popular. Furthermore, we are still a long way from mature solutions that for instance, can cope with multiple optimization criteria, and much work still needs to be done in the area of holistic system analysis/evaluation. We believe that the maturity level of adaptive systems research will rise as adaptive operation becomes a key requirement of protocol design rather than an "afterthought" or "add-on" functionality, as seen in many of the surveyed cases.

\section{REFERENCES}

Abolhasan, M., Wysocki, T., AND Dutkiewicz, E. 2004. A review of routing protocols for mobile ad hoc networks. Ad Hoc Networks 2, 1, 1-22.

Akyildiz, I. F., LeE, W.-Y., AND ChowdhuRY, K. R. 2009. Crahns: Cognitive radio ad hoc networks. Ad Hoc Networks 7, 5, 810-836.

AKYILDIZ, I. F., WANG, X., AND WANG, W. 2005. Wireless mesh networks: a survey. Computer Networks 47, $445-487$.

Amorim, M. D., Ziviani, A., Viniotis, Y., And Tassiulas, L. 2008. Practical aspects of mobility in wireless self-organizing networks (guest editorial). IEEE Wireless Communications Magazine 15, 6, 6-7.

ANA 2006. Autonomic network architecture. http://www.ana-project.org/web/.

Avancha, S., PAtel, C., AND Joshi, A. 2004. Ontology-driven adaptive sensor networks. In 1st Annual International Conference on Mobile and Ubiquitous Systems: Networking and Services (Mobiquitous). 194-202.

AWARE 2006. Platform for autonomous self-deploying and operation of wireless sensor-actuator networks cooperating with aerial objects. http://www.aware-project.net/.

BAHL, P. AND PADMANABHAN, V. N. 2000. RADAR: An in-building RF-based user location and tracking system. In 19th Annual Joint Conference of the IEEE Computer and Communications Societies (Infocom). Tel-Aviv, Israel.

Balasubramanian, A., Levine, B. N., And Venkaratamani, A. 2007. DTN routing as a resource allocation problem. In ACM SIGCOMM. 
BALZANO, L. AND NOWAK, R. 2007. Blind calibration of networks. In International Workshop on Information Processing in Sensor Networks (IPSN).

BARR, K. C. AND AsAnOviC, K. 2006. Energy-aware lossless data compression. ACM Transactions on Computer Systems 24, 3, 250-291.

Basagni, S., Carosi, A., Melachrinoudis, E., Petrioli, C., and Wang, Z. M. 2008. Controlled sink mobility for prolonging wireless sensor networks lifetime. ACM/Kluwer Wireless Networks 14, 6, 831858.

Basagni, S., Chlamtac, I., Syrotivk, V. R., AND Woodward, B. A. 1998. A distance effect algorithm for mobility (DREAM). In 4th Annual ACM/IEEE International Conference on Mobile Computing and Networking (MobiCom).

BASAgni, S., Turgut, D., AND DAS, S. K. 2001. Mobility-adaptive protocols for managing large ad hoc networks. In IEEE International Conference on Communications (ICC). 1539-1543.

Benbadis, F., DE Amorim, M. D., AND FdidA, S. 2005. Elip: Embedded location information protocol. In International IFIP-TC6 Networking Conference.

BICKET, J. 2005. Bit-rate selection in wireless networks. M.S. thesis, Massachusetts Institute of Technology.

Boonma, P. AND SUZUKI, J. 2007. Evolutionary constraint-based multi-objective adaptation for selforganizing wireless sensor networks. In Bio-inspired Models of Network, Information and Computing Systems (Bionetics). 111-119.

Borrel, V., Legendre, F., DE AMORIM, M. D., AND FDIDA, S. 2008. SIMPS: Using sociology for personal mobility. IEEE / ACM Transactions on Networking 17, 3, 831-842.

Buonadonna, P., Gay, D., J.Hellerstein, Hong, W., And Madden, S. 2005. Task: Sensor network in a box. Tech. rep., Technical Report IRB-TR-04-021, Intel Research Berkeley. January.

Burgess, J., Gallaghern, B., Jensen, D., And Levine, B. N. 2006. MaxProp: routing for vehicle-based disruption-tolerant networks. In 25th Annual Joint Conference of the IEEE Computer and Communications Societies (Infocom).

Burns, B., Brock, O., AND Levine, B. N. 2008. MORA routing and capacity building in disruptiontolerant networks. Ad Hoc Networks 6, 4, 600-620.

BurRi, N., von RickenBACH, P., WATtenhofer, R., AND Weber, Y. 2006. Topology control made practical: Increasing the performance of source routing. In 2nd International Conference on Mobile Ad-hoc and Sensor Networks (MSN).

Busnel, Y., Bertier, M., AND Kermarrec, A.-M. 2008. Solist or how to look for a needle in a haystack. a lightweight multi-overlay structure for wireless sensor networks. In 4th IEEE International Conference on Wireless and Mobile Computing, Networking and Communications (WiMob).

Busnel, Y., Querzoni, L., BALdoni, R., Bertier, M., AND KermarReC, A.-M. 2008. On the deterministic tracking of moving objects with a binary sensor network. In International Conference on Distributed Computing in Sensor Systems (DCOSS).

Bychkovskiy, V., Megerian, S., Estrin, D., And Potkonjak, M. 2003. Colibration: A collaborative approach to in-place sensor calibration. In International Workshop on Information Processing in Sensor Networks (IPSN).

CAI, H. AND EUN, D. Y. 2008. Toward stochastic anatomy of inter-meeting time distribution under general mobility models. In 9th ACM International Conference on Mobile Ad Hoc Networking and Computing (MobiHoc).

Cerpa, A. AND Estrin, D. 2004. Ascent: Adaptive self-configuring sensor networks topologies. IEEE Transactions on Mobile Computing 3, 3, 272-285.

Chaintreau, A., Hui, P., Crowcroft, J., Diot, C., Gass, R., And Scott, J. 2007. Impact of human mobility on the design of opportunistic forwarding algorithms. IEEE Transactions on Mobile Computing 6, 6, 600-620.

Chatzigiannakis, I., Kinalis, A., AND Nikoletseas, S. 2008. Efficient data propagation strategies in wireless sensor networks using a single mobile sink. Computer Communications 31, 5, 896-914.

Chen, B., Jamieson, K., Balakrishnan, H., AND MorRis, R. 2001. Span: An energy-efficient coordination algorithm for topology maintenance in ad hoc wireless networks. In 7th Annual ACM/IEEE International Conference on Mobile Computing and Networking (MobiCom). 85-96.

Chen, M. AND Fowler, M. L. 2005. Geometry-adaptive data compression for tdoa/fdoa location. In IEEE International Conference on Acoustics, Speech, and Signal Processing (ICASSP).

Cheng, C., Lemberg, H., Philip, S., van Den Berg, E., And Zhang, T. 2002. SLALoM: A scalable location management scheme for large mobile ad-hoc networks. In IEEE Wireless Communications and Networking Conference (WCNC).

Clausen, T. AND JACQUeT, P. 2003. Optimized link state routing protocol (OLSR). RFC 3626. 
ConAn, V., LegUAY, J., AND FRIEdman, T. 2007. Characterizing pairwise inter-contact patterns in delay tolerant networks. In International Conference on Autonomic Computing and Communication Systems (Autonomics).

Costa, P., Cesana, M., Brambilla, S., Casartelli, L., And Pizziniaco, L. 2008. A cooperative approach for topology control in wireless sensor networks: Experimental and simulation analysis. In International Symposium on a World of Wireless, Mobile and Multimedia Networks (WoWMoM).

Croce, S., Marcelloni, F., ANd Vecchio, M. 2008. Reducing power consumption in wireless sensor networks using a novel approach to data aggregation. The Computer Journal 51, 2, 1227-1239.

Dabek, F., Cox, R., KAAshoek, F., AND Morris, R. 2004. Vivaldi: A decentralized network coordinate system. In ACM SIGCOMM.

DALU, S. S., NASKAR, M. K., AND SARKAR, C. K. 2008. Implementation of a topology control algorithm for mobile ad hoc networks using pursue mobility model. Journal of Applied Computer Science 2, 2, 14-20.

DALY, E. AND HAAHR, M. 2007. Social network analysis for routing in disconnected delay-tolerant manets. In 8th ACM International Conference on Mobile Ad Hoc Networking and Computing (MobiHoc).

DAS, S. M., PUCH, H., AND HU, Y. C. 2005. Performance comparison of scalable location services for geogrphic ad hoc routing. In 24th Annual Joint Conference of the IEEE Computer and Communications Societies (Infocom).

Dobson, S., Denazis, S., Fernndez, A., Gati, D., Gelenbe, E., Massacci, F., Nixon, P., Saffre, F., SCHIMIDT, N., AND ZAMBONELLI, F. 2006. A survey of autonomic communications. ACM Transactions on Autonomous and Adaptive Systems (TAAS) 1, 2.

Drabkin, V., FRIEDMAN, R., KLIOT, G., AND SEGAL, M. 2007. RAPID: reliable probabilistic distribution in wireless ad-hoc networks. In 26th IEEE International Symposium on Reliable Distributed Systems (SRDS).

Dubois-Ferriere, H., Grossglauser, M., AND Vetterli, M. 2003. Age matters: Efficient route discovery in mobile ad hoc networks using encounter ages. In 4th ACM International Conference on Mobile Ad Hoc Networking and Computing (MobiHoc). Annapolis, MD.

EHF 2009. Energy harvesting forum. http://www.energyharvesting.net/.

Enge, P. And Misra, P. 1999. Special issue on global positioning system. Proceedings of the IEEE 87, 1, $3-15$.

Eriksson, J., Faloutsos, M., AND Krishnamurthy, S. 2004. Scalable ad hoc routing: The case for dynamic addressing. In 23th Annual Joint Conference of the IEEE Computer and Communications Societies (Infocom).

Erramilli, V., Crovella, M., Chaintreau, A., And Diot, C. 2008. Delegation forwarding. In 9th ACM International Conference on Mobile Ad Hoc Networking and Computing (MobiHoc).

Estrin, D., Govindan, R., Heidemann, J., AND Kumar, S. 1999. Next century challenges: Scalable coordination in sensor networks. In 5th Annual ACM / IEEE International Conference on Mobile Computing and Networking (MobiCom). Seattle, Washington, USA, 263-270.

FALL, K. 2004. A delay-tolerant network architecture for challenged internets. In ACM SIGCOMM.

FRANCOIS, J.-M. AND LEDUC, G. 2006. Predictable disruption tolerant networks and delivery guarantees. Tech. Rep. cs/0612034, ArXiv Computer Science e-prints. December.

Friedman, R., Kliot, G., AND Avin, C. 2008. Probabilistic quorum systems in wireless ad hoc networks. In 9th IEEE Conference on Dependable Systems and Networks (DSN).

Ganesan, D., Estrin, D., And Heidemann, J. 2002. Dimensions: Whay do we need a new data handling architecture for sensor networks? In ACM Workshop on Hot Topics in Networks (HotNets).

Garetto, M., Chiasserini, C. F., AND Leonardi, E. 2008. Sensor deployment and relocation: A unified scheme. Journal of Computer Science and Technology 23, 3, 400-412.

GeLenbe, E. 2009. Steps towards self-aware networks. Communications of the ACM 52, 7.

Gelenbe, E., Lent, R., And Xu, Z. 2001. Design and performance of cognitive packet networks. Performance Evaluation 46, 2-3, 155-176.

GioRDANO, S. AND HAMI, M. 1999. Mobility management: virtual home region. Tech. rep., Technical Report No. SSC/1999/037, EPFL. October.

Glance, N. ANd Snowdon, D. 2001. Pollen: using people as a communication medium. Elsevier Computer Networks 35, 4, 429-442.

Gomez, J. AND CAMPBELL, A. T. 2003. PARO: Supporting dynamic power controlled routing in wireless ad hoc networks. ACM / Kluwer Wireless Networks 9, 5, 443-460.

Gorcin, A. AND ARSLAN, H. 2008. Public safety and emergency case communications: Opportunities from the aspect of cognitive radio. In IEEE DySpan. 
Gross, T., Steenkiste, P., And Subhlok, J. 1999. Adaptive distributed applications on heterogenous networks. In Heterogenous Computing Workshop (HCW). 209-218.

Grossglauser, M. AND VetTerLi, M. 2003. Locating nodes with ease: Mobility diffusion of last encounters in ad hoc networks. In 22th Annual Joint Conference of the IEEE Computer and Communications Societies (Infocom).

Gupta, P. AND KUMAR, P. 2000. The capacity of wireless networks. IEEE Tansactions on Information Theory 46, 2, 388-404.

HAAS, Z. J. AND LIANG, B. 1999. Ad hoc mobility management with uniform quorum systems. IEEE/ACM Transactions on Networking 7, 2, 228-240.

HaAs, Z. J., Pearlman, M. R., AND SAMAR, P. 2002. The zone routing protocol (ZRP) for ad hoc networks. Internet-Draft.

Hamida, E. B. AND Chelius, G. 2008. Strategies for data dissemination to mobile sinks in wireless sensor networks. IEEE Wireless Communications 15, 6, 31-37.

HARRIS, A. F. 2006. Cross-layer energy-efficient mobile system design. Ph.D. thesis, University of Illinois at Urbana-Champaign.

HARTER, A. AND HopPer, A. 1994. A distributed location system for the active office. IEEE Network 8, 1, 62-70.

Havinga, P. J. M. AND SMit, G. J. M. 2001. Energy-efficient wireless networking for multimedia applications. Journal of Wireless Communications and Mobile Computing (WCMC), 165-184.

Heinzelman, W., Chandrakasan, A., AND Balakrishnan, H. 2000. Energy-efficient communication protocols for wireless microsensor networks. In Hawaaian International Conference on Systems Science (HICSS).

Heinzelman, W., Kulik, J., AND Balakrishnan, H. 1999. Adaptive protocols for information dissemination in wireless sensor networks. In 5th Annual ACM/IEEE International Conference on Mobile Computing and Networking (MobiCom). 174-185.

Hellerstein, J. M., Hong, W., Madden, S., And StaneK, K. 2003. Beyond average: Toward sophisticated sensing with queries. In International Workshop on Information Processing in Sensor Networks (IPSN).

Heylighen, F. 2001. The science of self-organization and adaptivity. In The Encyclopedia of Life Support Systems (EOLSS), L. D. Kiel, Ed. Eolss Publishers, Chapter Knowledge Management, Organizational Intelligence and Learning, and Complexity.

Holland, G., VAIDYA, N., AND BAHL, P. 2001. A rate-adaptive mac protocol for multi-hop wireless networks. In 7th Annual ACM / IEEE International Conference on Mobile Computing and Networking (MobiCom). 236-251.

Hong, X., Xu, K., AND Gerla, M. 2002. Scalable routing protocols for mobile ad hoc networks. IEEE Network 16, 4, 11-21.

HU, C. AND HOU, J. C. 2004. LISP: a link-indexed statistical traffic prediction approach to improving IEEE 802.11 PSM. In 24th IEEE International Conference on Distributed Computing Systems (ICDCS). 292 300.

HU, Y., WU, Z., AND GuO, M. 2007. Ontology driven adaptive data processing in wireless sensor networks. In 2nd International Conference on Scalable Information Systems (INFOSCALE).

Hubaux, J. P., Gross, T., Boudec, J. Y. L., And Vetterli, M. 2001. Towards self-organized mobile ad hoc networks: the terminodes project. IEEE Communications Magazine 39, 1, 118-124.

Huebscher, M. C. AND MCCANn, J. A. 2008. A survey on autonomic computing - degrees, models, and applications. Computing Surveys ACM 40, 3, 7-28.

Hui, P., Crowcroft, J., AND Yoneki, E. 2008. BUBBLE Rap: Social-based forwarding in delay tolerant networks. In 9th ACM International Conference on Mobile Ad Hoc Networking and Computing (MobiHoc).

IEEE 802.11 WG. Part 11: Wireless LAN medium access control (MAC) and physical layer (PHY) specifications, 1999 .

IEEE 802.16E-2005 AND IEEE STD 802.16-2004/COR1-2005. IEEE standard for local and metropolitan area networks part 16: Air interface for fixed and mobile broadband wireless access systems amendment 2: Physical and medium access control layers for combined fixed and mobile operation in licensed bands and corrigendum 1.

Intanagonwiwat, C., Govindan, R., And Estrin, D. 2000. Directed diffusion: a scalable and robust communication paradigm for sensor networks. In 6th Annual ACM/IEEE International Conference on Mobile Computing and Networking (MobiCom). 56-67. 
IOANNIDIS, S. AND MARBACH, P. 2005. Towards an understanding of ease and its properties. In International Symposium on Modeling and Optimization in Mobile, Ad Hoc, and Wireless Networks WiOpt.

JAIN, S., FALL, K., AND PATrA, R. 2004. Routing in a delay tolerant network. In ACM SIGCOMM.

Jea, D., Somasundara, A., And Srivastava, M. 2005. Multiple controlled mobile elements (data mules) for data collection in sensor networks. In International Conference on Distributed Computing in Sensor Systems (DCOSS).

Jelasity, M., Montresor, A., AND BABAoglu, O. 2005. Gossip-based aggregation in large dynamic networks. ACM Transactions on Computer Systems 23, 3, 219-252.

Johnson, D., HU, Y., AND MALTZ, D. 2007. The dynamic source routing protocol (DSR) for mobile ad hoc networks for IPv4. RFC 4728.

Kamerman, A. AND Monteban, L. 1997. Wavelan(c)-ii: a high-performance wireless lan for the unlicensed band. Bell Labs Technical Journal 2, 3, 118-133.

Kassabalidis, I., El-Sharkawi, M. A., Marks II, R. J., Arabshahi, P., and Gray, A. A. 2001. Swarm intelligence for routing in communication networks. In IEEE Global Communications Conference (GlOBECOM).

Kermarrec, A.-M., Mostéfaoui, A., Raynal, M., Trédan, G., and (alphabetical order), A. C. V. 2009. Large-scale networked systems: from anarchy to geometric self-structuring. In 10th International Conference on Distributed Computing and Networking (ICDCN).

Kiess, W., FÜssler, H., Widmer, J., AND MAUve, M. 2004. Hierarchical location service for mobile ad-hoc networks. ACM SIGMOBILE Mobile Computing and Communications Review (MC2R) 8, 4, 47-58.

Kim, D., Garcia-LunA-Aceves, J. J., ObRACZKA, K., CANO, J., AND MANZONI, P. 2003. Routing mechanims for mobile ad hoc networks based on the energy drain rate. IEEE Transactions on Mobile Computing 2, 2, 161-173.

KimurA, N. AND LATifi, S. 2005. A survey on data compression in wireless sensor networks. In International Conference on Information Technology.

Ko, Y.-B. AND VAIDYA, N. H. 2000. Location-aided routing (LAR) in mobile ad hoc networks. ACM/Kluwer Wireless Networks 6, 4, 307-321.

Kompella, R. R. ANd Snoeren, A. C. 2003. Practical lazy scheduling in sensor networks. In 1st International Conference on Embedded Networked Sensor Systems (SenSys). 280-291.

KRASHINSKY, R. AND BALAKRISHNAN, H. 2005. Minimizing energy for wireless web access with bounded slowdown. ACM/Kluwer Wireless Networks 11, 1-2, 135-148.

Krifa, A., BArakat, C., AND Spyropoulos, T. 2008. Optimal buffer management policies for delay tolerant networks. In IEEE Communications Society Conference on Sensor and Ad Hoc Communications and Networks (SECON).

KRUnZ, M., MUQATTASH, A., AND LEE, S.-J. 2004. Transmission power control in wireless ad hoc networks: Challenges, solutions, and open issues. IEEE Network 8, 15, 8-14.

KUliK, J., HEINZELMAN, W., AND BALAKRISHNAN, H. 2002. Negotiation-based protocols for disseminating information in wireless sensor networks. ACM / Kluwer Wireless Networks 8, 169-185.

LACAge, M., MANShaei, M. H., AND TURLetTi, T. 2004. Ieee 802.11 rate adaptation: a practical approach. In 7th ACM international symposium on Modeling, analysis and simulation of wireless and mobile systems (MSWIM). 126-134.

Lamarca, A., Brunette, W., Koizumi, D., Lease, M., Sigurdsson, S. B., Sikorski, K., Fox, D., AND BORRIELLO, G. 2002. Making sensor networks practical with robots. Tech. rep., Technical Report IRS-TR-02-004, Intel Research. January.

Li, J., Jannotti, J., Couto, D. S. J. D., KARger, D. R., AND MorRis, R. 2000. A scalable location service for geographic ad hoc routing. In 6th Annual ACM / IEEE International Conference on Mobile Computing and Networking (MobiCom).

Li, X., Santora, N., AND Stojmenovic, I. 2007. Mesh-based sensor relocation for coverage maintenance in mobile sensor networks. In Ubiquitous Intelligence and Computing.

Lilien, L., Gupta, A., AND YANG, Z. 2007. Standard implementation framework for opportunistic networks in emergency preparedness and response applications. In International Workshop on Research Challenges in Next Generation Networks for First Responders and Critical Infrastructures (NetCri07).

Lilien, L., KAmAL, Z., Bhuse, V., AND A.GUPTA. 2006. Opportunistic networks: The concept and research challenge. In International Workshop on Research Challenges In Security and Privacy for Mobile and Wireless Networks (WSPWN 06).

Lilien, L., Kamal, Z. H., Gupta, A., Bhuse, V., AND Yang, Z. 2006. Opportunistic sensor networks (oppnets). In Third International Conference on Networked Sensing Systems (INSS'06). 
Lindgren, A., Diot, C., AND ScotT, J. 2006. Impact of communication infrastructure on forwarding in pocket switched networks. In ACM SIGCOMM.

Lindgren, A., Doria, A., AND Schelen, O. 2003. Probabilistic routing in intermittently connected networks. ACM SIGMOBILE Mobile Computing and Communications Review (MC2R) 7, 3, 19-20.

LiU, D., Stojmenovic, I., AND X.JiA. 2008. A scalable quorum-based location service in ad hoc and sensor networks. International Journal of Communication Networks and Distributed Systems 1, 1.

LU, G., KRIShNAMAChaRI, B., AND RAghavendRA, C. S. 2007. An adaptive energy-efficient and lowlatency MAC for tree-based data gathering in sensor networks. Journal of Wireless Communications and Mobile Computing (WCMC) 7, 7, 863-875.

LUO, H., LUO, J., LIU, Y., AND DAS, S. K. 2006. Adaptive data fusion for energy efficient routing in wireless sensor networks. IEEE Transactions on Computers 55, 10, 1286-1299.

Luo, H., Ye, F., Cheng, J., Lu, S., AND Zhang, L. 2005. TTDD: Two-tier data dissemination in large-scale wireless sensor networks. ACM / Kluwer Wireless Networks 11, 1, 161-175.

Luo, J., Panchard, J., Piorkowski, M., Grossglauser, M., And Hubaux, J. P. 2006. Mobiroute: Routing towards a mobile sink for improving lifetime in sensor networks. In International Conference on Distributed Computing in Sensor Systems (DCOSS). 480-497.

MAinland, G., PARKES, D. C., AND Welsh, M. 2005. Decentralized, adaptive resource allocation for sensor networks. In 2 nd Symposium on Networked Systems Design and Implementation (NSDI). 315-328.

MAJID, S. AND Ahmed, K. 2008. Post-disaster communications: A cognitive agent approach. In Seventh International Conference on Networking.

MaKram, S. A., GunEs, M., KCHICHE, A., AND KreBs, M. 2008. Dynamic channel assignment for wireless mesh networks using clustering. In IEEE International Conference on Networking (ICN). 539-544.

MARCELlONI, F. AND VECCHIO, M. 2008. A simple algorithm for data compression in wireless sensor networks. IEEE Communications Letters 12, 6.

Marcelloni, F. AND VeCChio, M. 2009. An efficient lossless compression algorithm for tiny nodes of monitoring wireless sensor networks. The Computer Journal 52, 9.

Massoulie, L., Merrer, E. L., Kermarrec, A.-M., And Ganesh, A. 2006. Peer counting and sampling in overlay networks: random walk methods. In ACM Symposium on Principles of Distributed Computing (PODC). 123-132.

Merrer, E. L., Gramoli, V., Viana, A. C., AND Bertier, M. 2006. Energy aware self-organizing density management in wireless sensor networks. In International Workshop on Decentralized Resource Sharing in Mobile Computing and Networking (ACM Mobishare). 24-29.

Miluzzo, E., Lane, N. D., Campbell, A. T., And Olfati-Saber, R. 2008. Calibree: a self-calibration system for mobile sensor networks. In International Conference on Distributed Computing in Sensor Systems (DCOSS).

Mirhakkak, M., Schult, N., And Thomson, D. 2001. Dynamic bandwidth management and adaptive applications for a variable bandwidth wireless environment. IEEE Journal on Selected Areas in Communications 19, 10, 1984-1997.

Mitola, J. AND MaguiRe, G. Q. 1999. Cognitive radio: Making software radios more personal. IEEE Personal Communications 6, 4, 13-18.

MtibaA, A., Chaintreau, A., AND Diot, C. 2006. Detail characterization of paths in pocket switched networks. In ACM Conference on Emerging Networking Experiments and Technologies (CoNEXT).

Nikaein, N., Laboid, H., AND Bonnet, C. 2000. Distributed dynamic routing algorithm (DDR) for mobile ad hoc networks. In 1st ACM International Conference on Mobile Ad Hoc Networking and Computing (MobiHoc).

OGier, R., Templin, F., AND Lewis, M. 2004. Topology dissemination based on reverse-path forwarding (TBRPF). RFC 3684.

PARK, S.-J. AND SivAKUmAR, R. 2003. Adaptive topology control for wireless ad hoc networks. ACM SIGMOBILE Mobile Computing and Communications Review (MC2R) 7, 3, 37-38.

PARK, V. D. AND CoRson, M. S. 1997. A highly adaptive distributed routing algorithm for mobile wireless networks. In 16th Annual Joint Conference of the IEEE Computer and Communications Societies (Infocom).

Perkins, C., BELding-Royer, E., AND DAs, S. 2003. Ad hoc on-demand distance vector (AODV) routing. RFC 3561.

Poellabauer, C. AND SCHWAN, K. 2004. Energy-aware traffic shaping for wireless real-time applications. In 10th IEEE Real-Time and Embedded Technology and Applications Symposium (RTAS).

Polastre, J., Hill, J., AND Culler, D. 2004. Versatile low power media access for wireless sensor networks. In 2 nd International Conference on Embedded Networked Sensor Systems (SenSys). 95-107. 
Prehofer, C. And Bettstetter, C. 2005. Self-organization in communication networks: principles and design paradigms. IEEE Communications Magazine 43, 7, 78-85.

Ramachandran, K. N., Belding, E. M., Almeroth, K. C., ANd Buddhikot, M. M. 2006. Interferenceaware channel assignment in multi-radio wireless mesh networks. In 25th Annual Joint Conference of the IEEE Computer and Communications Societies (Infocom).

Ramanathan, R., Hansen, R., Basu, P., Rosales-Hain, R., And Krishnan, R. 2007. Prioritized epidemic routing for opportunistic networks. In 1st International Workshop on Mobile Opportunistic Networking (MobiOpp).

Ratnasamy, S., Estrin, D., Govindan, R., Karp, B., And Shenker, S. 2002. Data-centric storage in sensornets. In ACM SIGCOMM.

Rokonuzzaman, S. M., Pose, R., ANd Gondal, I. 2008. A framework for a qos based adaptive topology control system for wireless ad hoc networks with multibeam smart antennas. In International Symposium on Parallel and Distributed Processing with Applications (ISPA).

RUNES 2006. Reconfigurable ubiquitous networked embedded systems. http://www.ist-runes.org/.

SAdeghi, B., Kanodia, V., SAbharwal, A., AND Knightly, E. 2005. OAR: an opportunistic auto-rate media access protocol for ad hoc networks. ACM / Kluwer Wireless Networks 11, 1-2, 39-53.

SAdler, C. M. AND Martonosi, M. 2006. Data compression algorithms for energy-constrained devices in delay tolerant networks. In 4th International Conference on Embedded Networked Sensor Systems (SenSys).

Schurgers, C. AND SRIVAstava, B. M. 2002. Energy-efficient wireless scheduling: Adaptive loading in time. In IEEE Wireless Communications and Networking Conference (WCNC). 706-711.

Schurgers, C., Tsiatsis, V., AND SRIVAstava, M. B. 2002. STEM: Topology management for energy efficient sensor networks. In IEEE Aerospace Conference.

SeEt, B.-C., PAN, Y., HsU, W.-J., AND LAU, C.-T. 2005. Multi-home region location service for wireless ad hoc networks: An adaptive demand-driven approach. In 2 nd Annual Conference on Wireless OnDemand Network Systems and Services (WONS). 258-263.

Sengul, C., Bakht, M., Harris, A. F., Abdelzaher, T., and Kravets, R. 2008. Improving energy conservation using bulk transmission over high-power radios in sensor networks. In 28th IEEE International Conference on Distributed Computing Systems (ICDCS).

Sengul, C., Harris, A. F., AND Kravets, R. 2007. Reconsidering power-management. In 4th International Conference on Broadband Communications, Networks, and Systems (Broadnets). Invited paper.

Sengul, C. AND KRAVETS, R. 2005. Conserving energy with on-demand topology management. In 2nd IEEE International Conference on Mobile Ad hoc and Sensor Systems (MASS).

SENGUL, C. AND KRAVETS, R. 2007. Heuristic approaches to energy-efficient network design problem. In 27th IEEE International Conference on Distributed Computing Systems (ICDCS).

Sengul, C., Miller, M., AND Gupta, I. 2008. Adaptive probability-based broadcast forwarding in energysaving sensor networks. ACM Transactions on Sensor Networks 4, 2.

Sengul, C., Viana, A. C., Friedman, R., Bertier, M., And Kermarrec, A.-M. 2009. The importance of being adaptive for forwarding. In Extreme Workshop on Communication (Extremecom).

Shah, S. S., Chen, K., AND NAhrstedt, K. 2005. Dynamic bandwidth management in single-hop ad hoc wireless networks. ACM / Kluwer Mobile Networks and Applications 10, 1-2, 199-217.

Shen, C.-C., HuAnG, Z., AND JAIKAEO, C. 2005. Ant-based distributed topology control algorithms for mobile ad hoc networks. ACM / Kluwer Wireless Networks 11, 3, 299-317.

SOCRATES 2007. Self-optimisation and self-configuration in wireless networks. http://www.fp7socrates.eu/.

Solis, I. AND OBRACZKA, K. 2005. Efficient continuous mapping in sensor networks using isolines. In $2 n d$ Annual International Conference on Mobile and Ubiquitous Systems: Networking and Services (Mobiquitous).

Spyropoulos, T., Psounis, K., And Raghavendra, C. 2008. Efficient routing in intermittently connected mobile networks: The single-copy case. IEEE / ACM Transactions on Networking 16, 1, 63-76.

Spyropoulos, T., Psounis, K., AND RAGHavendRA, C. S. 2007. Spray and focus: Efficient mobilityassisted routing for heterogeneous and correlated mobility. In IEEE International Conference on Pervasive Computing and Communications Workshops.

Stojmenovic, I. 2002. Location updates for efficient routing in ad hoc networks. In Handbook of wireless networks and mobile computing. 451-471.

SU, J., ChIn, A., GoEL, A. P. A., AND DE LARA, E. 2004. User mobility for opportunistic ad-hoc networking. In IEEE Workshop on Mobile Computing Systems and Applications (WMCSA). 
SU, J., Goel, A., AND DE LARA, E. 2006. An empirical evaluation of the student-net delay tolerant network. In 3rd Annual International Conference on Mobile and Ubiquitous Systems: Networking and Services (Mobiquitous).

Talebi, M. S., Kefayati, M., Khalaj, B. H., And Rabiee, H. R. 2006. Adaptive consensus averaging for information fusion over sensor networks. In 3rd IEEE International Conference on Mobile Ad hoc and Sensor Systems (MASS).

ToH, C. K. 2001. Maximum battery life routing to support ubiquitous mobile computing in wireless ad hoc networks. IEEE Communications Magazine 39, 6, 138-147.

Tseng, Y.-C., Ni, S.-Y., Chen, Y.-S., AND SheU, J.-P. 2002. The broadcast storm problem in a mobile ad hoc network. ACM/Kluwer Wireless Networks 8, 2/3, 153-167.

TSEnG, Y.-C., NI, S.-Y., AND SHIH, E.-Y. 2003. Adaptive approaches to relieving broadcast storms in a wireless multihop mobile ad hoc network. IEEE Transactions on Computers 52, 5, 545-557.

URGAONKAR, R. AND KRISHNAMACHARI, B. 2004. Flow: An efficient forwarding scheme to mobile sink in wireless sensor networks. In IEEE Communications Society Conference on Sensor and Ad Hoc Communications and Networks (SECON).

Uysal-Biyikoglu, E., Prabhakar, B., And Gamal, A. E. 2002. Energy-efficient packet transmission over a wireless link. IEEE / ACM Transactions on Networking 10, 4, 487-500.

VAHDAT, A. AND BECKER, D. 2000. Epidemic routing for partially-connected ad hoc networks. Tech. rep., Duke University.

VAn DAM, T. AND LANGendoen, K. 2003. An adaptive energy-efficient mac protocol for wireless sensor networks. In 1st International Conference on Embedded Networked Sensor Systems (SenSys). 171-180.

Vecchio, M., Viana, A. C., Ziviani, A., AND Friedman, R. 2010. DEEP: Density-based proactive data dissemination protocol for wireless sensor networks with uncontrolled sink mobility. Computer Communications 33, 8, 929-939.

Viana, A. C., Amorim, M. D., FdidA, S., AND Rezende, J. F. 2005. Self-organization in spontaneous networks: the approach of DHT-based routing protocols. Ad Hoc Networks 3, 5, 589-606.

VianA, A. C., Amorim, M. D., Viniotis, Y., FdidA, S., AND Rezende, J. F. 2006. Twins: a dual addressing space representation for self-organizing networks. IEEE Transactions on Parallel and Distributed Systems 17, 12, 1468-1481.

Viana, A. C., Ziviani, A., And Friedman, R. 2009. Decoupling data dissemination from mobile sink's trajectory in wireless sensor networks. IEEE Communications Letters 13, 3, 178-180.

WANG, G., CAO, G., AND PorTA, T. F. L. 2006. Movement-assisted sensor deployment. 5, 6, 640-652.

WANG, G., CAO, G., Porta, T. F. L., AND ZhanG, W. 2005. Sensor relocation in mobile sensor networks. In 24th Annual Joint Conference of the IEEE Computer and Communications Societies (Infocom). 23022312.

WANG, Y. AND WU, H. 2006. Dft-msn: The delay/fault-tolerant mobile sensor network for pervasive information gathering. In 25th Annual Joint Conference of the IEEE Computer and Communications Societies (Infocom).

Woo, S.-C. M. AND SINGH, S. 2001. Scalable routing protocol for ad hoc networks. ACM/Kluwer Wireless Networks 7, 5, 513-529.

Xu, K., TAng, K., Bagradio, R., Gerla, M., And Bereschinsky, M. 2003. Adaptive bandwidth management and QoS provisioning in large scale ad hoc networks. In IEEE Military Communications Conference (MILCOM). Vol. 2. 1018-1023.

XU, Y., Heidemann, J., AND Estrin, D. 2000. Adaptive energy-conserving routing for multihop ad hoc networks. Tech. rep., USC Information Sciences Institue.

Xue, Y., CUI, Y., AND NAhrstedt, K. 2005. Maximizing lifetime for data aggregation in wireless sensor networks. ACM / Kluwer Mobile Networks and Applications 10, 6, 853-864.

Xue, Y., LI, B., AND NAhrstedt, K. 2001. A scalable location management scheme in mobile ad-hoc networks. In 26th Annual IEEE Conference on Local Computer Networks (LCN).

YANG, H., YE, F., AND SikdaR, B. 2006. SIMPLE: Using swarm intelligence methodology to design data acquisition protocol in sensor networks with mobile sinks. In 25th Annual Joint Conference of the IEEE Computer and Communications Societies (Infocom).

YE, W., HEIDEMANN, J., AND EstRIN, D. 2002. An energy-efficient MAC protocol for wireless sensor networks. In 21st Annual Joint Conference of the IEEE Computer and Communications Societies (Infocom). 1567-1576.

Ye, W., Silva, F., AND Heidemann, J. 2006. Ultra-low duty cycle MAC with scheduled channel polling. In 4th International Conference on Embedded Networked Sensor Systems (SenSys). 321-334. 
Younis, O., Kant, L., Chang, K., AND Young, K. 2009. Cognitive manet design for mission-critical networks. IEEE Communications Magazine 47, 10.

Zhang, A., Kokkeler, A. B. J., AND SMit, G. J. M. 2006. A reconfigurable radio architecture for cognitive radio in emergency networks. In Proceedings of the 9th European Conference on Wireless Technology.

Zhao, W., Ammar, M., And Zegura, E. 2004. A message ferrying approach for data delivery in sparse mobile ad hoc networks. In 5th ACM International Conference on Mobile Ad Hoc Networking and Computing (MobiHoc).

Zhao, W., Chen, Y., Ammar, M., Corner, M., Levine, B., AND Zegura, E. 2006. Capacity enhancement using throwboxes in dtns. In 3rd IEEE International Conference on Mobile Ad hoc and Sensor Systems (MASS).

Zheng, R., Hou, J. C., AND SHA, L. 2003. Asynchronous wakeup for ad hoc networks. In 4th ACM International Conference on Mobile Ad Hoc Networking and Computing (MobiHoc). 35-45.

Zheng, R. AND KRAVets, R. 2003. On-demand power management for ad hoc networks. In 22th Annual Joint Conference of the IEEE Computer and Communications Societies (Infocom).

ZHENG, T., RADHAKRISHNAN, S., AND SARANGAN, V. 2005. PMAC: an adaptive energy-efficient mac protocol for wireless sensor networks. In 19th IEEE International Parallel and Distributed Processing Symposium (IPDPS). 